\title{
NORI DIAGRAMS AND PERSISTENT HOMOLOGY
}

\author{
Yuri I. Manin, Matilde Marcolli \\ ${ }^{1}$ Max-Planck-Institut für Mathematik, Bonn, Germany, \\ ${ }^{2}$ California Institute of Technology, Pasadena, USA \\ University of Toronto, Canada \\ Perimeter Institute for Theoretical Physics, Canada
}

\begin{abstract}
Recently, it was found that there is a remarkable intuitive similarity between studies in theoretical computer science dealing with large data sets on the one hand, and categorical methods of topology and geometry in pure mathematics, on the other. In this article, we treat the key notion of persistency from computer science in the algebraic geometric context involving Nori motivic constructions and related methods. We also discuss model structures for persistent topology.
\end{abstract}

\section{INTRODUCTION AND SUMMARY}

This paper is a contribution to the emerging research field in which computational mathematics dealing with large data bases interacts with topology, homological algebra, and "brave new algebra" of homotopy theory.

As a remarkable result of such interaction, (various versions of) the notion of persistent homology appeared. According to the informative survey presented in [BuSiSc15], the general notion of persistence in computational mathematics was informed by the topological data analysis. For a general introduction and overview of persistent homology and topological data analysis, see [BoChYv18], [Car09], [EdHar10], [Za05].

Large data bases are first represented by a family of sampled data at various scales. Then each sample is structured as a topological/algebraic object say, a simplicial space or its chain complex, which are interrelated by a nesting relation. Finally, the invariants of these objects at all scales are compared, so that those of them that are persistent across a sufficiently large range of scales become encoded in a persistence diagram, or barcode. An intuitively transparent picture of this kind, 
leading to a multidimensional bar-encoding of derived category of sheaves on a real finite-dimensional vector space, was developed very recently in [KaSch17], while to our knowledge the earliest introduction of persistence and bar code diagrams, in the topological/homotopical context, is due to S. Barannikov, [Bar94].

Our starting point was the observation that conversely, some of the important and already well formalised technical tools of algebraic topology and algebraic geometry may be represented as the product of intuitive search for "persistent" properties of topological spaces/ algebraic varieties/schemes "observed" at various scales or from various distances.

In this paper, we focus on the formalism of Nori diagrams and Nori motives (see [HuM-S17] and [Ar13]) and show that persistence philosophy presents them in a new light.

In mathematical community, existence of a rich ramification of persistency ideas is not as widely known as it deserves. Hence we hope that our input might be fruitful.

0.1. Diagrams in various contexts. We work below in a fixed small universe as it was presented in [KaSch06], Ch. 1. We do not mention the universe explicitly.

0.1.1. Definitions. ([HuM-St17], Def. 7.1.1) A diagram $D$ is a family, consisting of two disjoint sets $V(D)$ (vertices), $E(D)$ (edges), and a map $\partial: E(D) \rightarrow$ $V(D) \times V(D), \partial(e)=\left(\partial_{\text {out }}(e), \partial_{\text {in }}(e)\right)$ (orientation of edges). An oriented edge is sometimes called an arrow.

Morphism of diagrams $D_{1} \rightarrow D_{2}$ consists of two maps $V\left(D_{1}\right) \rightarrow V\left(D_{2}\right), E\left(D_{1}\right) \rightarrow$ $E\left(D_{2}\right)$, compatible with orientations.

A diagram with identities is a diagram $D$ in which for every vertex $v$, exactly one oriented edge from $v$ to $v$ is given and called the identity edge $i d_{v}$. Morphism of diagrams with identities must map identities to identities.

For example, each category $\mathcal{C}$ defines a diagram with identities $D(\mathcal{C})$ for which

$$
V(D(\mathcal{C})):=\operatorname{Ob} \mathcal{C}, \quad E(D(\mathcal{C})):=\operatorname{Hom} \mathcal{C}
$$

and $\partial(f: X \rightarrow Y):=(X, Y)$.

Given a diagram $D$ and a category $\mathcal{H}$, any morphism of diagrams $D \rightarrow D(\mathcal{H})$ is called a representation of $D$. Of course, representations (perhaps, satisfying additional compatibility conditions) themselves are objects of a category/ vertices 
of its diagram etc. This is the universe where various persistence intuitions reside and constructions of persistence invariants develop.

We will start here with a brief description of persistence constructions developed in computer science, and then give a short survey of Nori's persistency.

0.2. Thin categories and diagrams. Let $(\mathcal{S}, \leq)$ be a poset that is, a set $\mathcal{S}$ with reflexive, transitive, and anti-symmetric binary order relation ([KaSch06], Def. 1.1.3.) It defines a diagram $D$ for which $V(D):=\mathcal{S}, E(D):=$ the set of all pairs $\left(s_{1}, s_{2}\right)$ such that $s_{1}<s_{2}$, oriented from $s_{1}$ to $s_{2}$.

0.2.1. Definition. A category $\mathcal{C}$ is called thin, if each set $\operatorname{Hom}(X, Y)$ consists of $\leq 1$ element.

Clearly, for such a category $\mathrm{ObC}$ has the canonical structure of a poset: $X<Y$ iff $X \neq Y$ and $\operatorname{Hom}(X, Y)$ is non-empty. Conversely, each poset defines in this way a thin category in which morphisms in $\operatorname{Hom}(X, Y)$ are equivalence classes of oriented paths from $X$ to $Y$. Hence, describing a thin category, one may restrict oneself to an explicit description of only generating morphisms and keep in mind that each diagram in a thin category is automatically commutative. Basic examples of posets/thin categories used in data mining are natural numbers $\mathbf{N}$ and real numbers R.

Let now $\mathcal{I}$ be a category. Then the functors $\mathcal{C} \rightarrow \mathcal{I}$ from a fixed category $\mathcal{C}$ to $\mathcal{I}$ form objects of a category denoted $\mathcal{I}^{\mathcal{C}}$, with natural transformations as morphisms. If "the indexing category" $\mathcal{I}$ is thin, then $\mathcal{I}^{\mathcal{C}}$ is also thin. More precisely, a natural transformation $F \rightarrow G$ exists if and only if $F(X) \leq G(X)$ for all $X \in \mathrm{ObC}$, and this last relation makes from $\mathcal{I}^{\mathcal{C}}$ a poset.

This remark allows one to define a general analog of the semigroup of oriented translations of the poset $\mathbf{R}: x \mapsto x+a$, for arbitrary thin category $\mathcal{I}$. Namely, it is the monoid $\operatorname{Trans}_{\mathcal{I}}:=\mathcal{I}^{\mathcal{I}}$ with respect to the composition. It acts on any $\mathcal{I}^{\mathcal{C}}$ by the precomposition. According to [BuSiSc15], p. 1511, "we can think of Trans as a sort of 'positive cone' in the monoid of all endomorphism (i. e., monotone functions) $\mathcal{I} \rightarrow \mathcal{I}$."

0.2.2. Example. Spectral Sequences. Our exposition below is based upon [GeMa03], pp. 200-218.

Let $r \geq 1$ be an integer. We will call the $r$-th page of a spectral sequence the following thin indexing category $\mathcal{E}_{r}$ :

$$
\operatorname{Ob} \mathcal{E}_{r}:=\text { triples }(p, q, r) \text {, where } p, q \in \mathbf{Z} \text {. }
$$


Besides identities, a system of generating morphisms of $\mathcal{E}_{r}$ consists of the arrows

$$
d_{r}^{p, q}:(p, q, r) \rightarrow(p+r, q-r+1, r) .
$$

Moreover, the last page of a spectral sequence is the thin category $\mathcal{E}_{\infty}$ whose objects are all pairs $(p, q) \in \mathbf{Z}^{2}$, and morphisms are generated by the arrows $(p, q) \rightarrow$ $(p+1, q-1)$.

Let now $\mathbf{A}$ be an additive category, and $F: \mathcal{E}_{r} \rightarrow \mathbf{A}$ be any functor, satisfying the additional condition

$$
F\left(d_{r}^{p, q}\right) \circ F\left(d_{r}^{p+r, q-r+1}\right)=0 \text { for all } p, q .
$$

Such functors form a thin subcategory of $\mathcal{E}_{r}^{\mathbf{A}}$ an object of which may be called the $r$-th page of an $\mathbf{A}$-valued spectral sequence.

Similarly, for the last page we consider the $\mathbf{A}$-valued functors $\mathcal{E}_{\infty} \rightarrow \mathbf{A}$ transforming each morphism $(p, q) \rightarrow(p+1, q-1)$ into the embedding

$$
F i l^{p+1} A^{n} \rightarrow F i l^{p} A^{n} \text { for } n=p+q
$$

where $F i l^{*}$ is family of filtrations on each object of a sequence of objects $A^{n}, n \in \mathbf{Z}$, in $\mathbf{A}$.

0.3. Role of thin diagrams in persistence constructions. Chronologically early definition of persistence module was a family of vector spaces $V_{s}$, indexed by $s \in \mathbf{N}$ or by $s \in \mathbf{R}$, endowed with a family of morphisms $f_{s, t}: V_{s} \rightarrow V_{t}$ whose properties can be succinctly expressed by the statement that this family forms a functor from the indexing category $\mathbf{N}$ or $\mathbf{R}$ to the target category of vector spaces. More generally, one can consider functors with values in a thin category such as pages of a spectral sequence.

0.3.1. Example. Sublevelset persistence module. It is a real valued function (say, piecewise continuous) on a topological space $f: X \rightarrow \mathbf{R}$ considered as a functor $F \in \mathbf{T o p}^{\mathbf{R}}, F: t \mapsto f^{-1}(-\infty, t] \subseteq X$.

Sublevelset persistence homology of $f$ is defined as a postcomposition of $F$ and a homology theory. One can consider points in the indexing diagram $s \in \mathbf{N}$ or $s \in \mathbf{R}$ at which persistence homology jumps up or down when we increase $t$, say $t^{+}$ or $t^{-}$. The resulting sequence of indexed numbers, together with some additional 
information about appearing/vanishing homology spaces, is called the barcode of this persistent homology. For more details, see Sec. 1.3 below.

We omit here an essential construction of interleaving distance. It was analysed in categorical terms in [BuSiSc15]. More precisely, the authors have shown that an interleaving distance can be defined by comparing the monoid $\operatorname{Trans}_{\mathcal{I}}$ with the monoid $[0, \infty]$ by a sublinear projection $\omega: \operatorname{Trans}_{\mathcal{I}} \rightarrow[0, \infty]$ : or with the monoid $[0, \infty)$ by a superlinear family $\Omega:[0, \infty) \rightarrow \operatorname{Trans}_{\mathcal{I}}$. Moreover, the authors observe that $\omega$ and $\Omega$ are dual in a precise categorical sense. From this observation, many of their properties follow easily. Then it becomes clear what is needed to replace the monoids $[0, \infty]$ and $[0, \infty)$ in order to obtain other ways of measuring interleavings. Comparing Transp with $[0, \infty]^{n}$ and $[0, \infty)^{n}$ the authors of [BuSiSc15] show that the resulting 'vector persistence' is stable.

0.4. Linear representations of diagrams and Nori's persistence: basic constructions. Start with the following data:

a) a diagram $D$;

b) a noetherian commutative ring with unit $R$ and the category of finitely generated $R$-modules $R-M o d$;

c) a representation $T$ of $D$ in $R-M o d$.

Let $\operatorname{End}(T)$ be defined as the ring

$\operatorname{End}(T):=\left\{\left(\phi_{v}\right) \prod_{v \in V(D)} \operatorname{End}_{R}(T(v)) \mid \phi_{\partial_{\text {out }}(e)} \circ T(e)=T(e) \circ \phi_{\partial_{\text {in }}(e)}, \forall e \in E(D)\right\}$

An inclusion of diagrams $D_{1} \subset D_{2}$ such that $T_{1}=\left.T_{2}\right|_{D_{1}}$ determines a homomorphism $\operatorname{End}\left(T_{2}\right) \rightarrow \operatorname{End}\left(T_{1}\right)$, by projecting the product $\prod_{v \in V\left(D_{2}\right)} \operatorname{End}_{R}\left(T_{2}(v)\right)$ onto the product $\prod_{v \in V\left(D_{1}\right)} \operatorname{End}_{R}\left(T_{1}(v)\right)$.

Produce from the data above the category $C(D, T)$ defined in the following way:

d1) If $D$ is finite, then $C(D, T)$ is the category $\operatorname{End}(T)$-Mod of finitely generated $R$-modules equipped with an $R$-linear action of $\operatorname{End}(T)$.

d2) If $D$ is infinite, first consider its all finite subdiagrams $F$.

For each $F$ construct $C\left(F,\left.T\right|_{F}\right)$ as in $\left.\mathrm{d} 1\right)$. Then apply the following limiting procedure. Objects of $C(D, T)$ will be all objects of the categories $C\left(F,\left.T\right|_{F}\right)$. If 
$F \subset F^{\prime}$, then each object $X_{F}$ of $C\left(F,\left.T\right|_{F}\right)$ gives an object of $X_{F^{\prime}}$ of $C\left(F^{\prime},\left.T\right|_{F^{\prime}}\right)$, via the map from $\operatorname{End}\left(T_{F}\right)$-Mod to $\operatorname{End}\left(T_{F^{\prime}}\right)$-Mod determined by the morphism $\operatorname{End}\left(T_{F^{\prime}}\right) \rightarrow \operatorname{End}\left(T_{F}\right)$ as above. Morphisms from $X$ to $Y$ in $C(D, T)$ will be defined as colimits over $F$ of morphisms from $X_{F}$ to $Y_{F}$ with respect to these extensions.

The result is called the diagram category $C(D, T)$. It is an $R$-linear abelian category which is endowed with $R$-linear faithful exact forgetful functor

$$
f_{T}: C(D, T) \rightarrow R-M o d
$$

For more details, see [HuM-S17], pp. 140-144.

0.4.1. Universal properties of diagram categories. Any representation $T: D \rightarrow R-M o d$ can be presented as precomposition of the forgetful functor $f_{T}$ with an appropriate representation $\tilde{T}: D \rightarrow C(D, T)$ :

$$
T=f_{T} \circ \tilde{T}
$$

with the following universal property:

Given any $R$-linear abelian category $A$ with a representation $F: D \rightarrow A$ and $R$ linear faithful exact functor $f: A \rightarrow R-\operatorname{Mod}$ with $T=f \circ F$, it factorises through a faithful exact functor $L(F): C(D, T) \rightarrow A$ compatibly with decomposition

$$
T=f_{T} \circ \tilde{T}
$$

For proofs, cf. [HuM-S17], pp. 140-141.

0.4.2. Persistence. The functor $L(F)$ is actually unique up to unique isomorphism of exact additive functors ([HuM-S17], p. 167). It is this functor, constructed for various diagrams of geometric origin in algebraic geometry/topology/... that is an embodiment of persistency in our context. Below we give a sketch of relevant constructions; their development in various geometric environments is the content of Sec. 1 of our paper.

0.5. Nori geometric diagrams. If we have a "geometric" category $\mathcal{C}$ of spaces/varieties/schemes, possibly endowed with additional structures, in which one can define morphisms of closed embeddings $Y \hookrightarrow X$ (or $Y \subset X$ ) and morphisms of complements to closed embeddings $X \backslash Y \rightarrow X$, we can define the Nori diagram of effective pairs $D(\mathcal{C})$ in the following way (see [HuM-S17], pp. 207-208). 
a). One vertex of $D(\mathcal{C})$ is a triple $(X, Y, i)$ where $Y \hookrightarrow X$ is a closed embedding, and $i$ is an integer.

b). Besides obvious identities, there are edges of two types.

b1). Let $(X, Y)$ and $\left(X^{\prime}, Y^{\prime}\right)$ be two pairs of closed embeddings. Every morphism $f: X \rightarrow X^{\prime}$ such that $f(Y) \subset Y^{\prime}$ produces functoriality edges $f^{*}$ (or rather $\left(f^{*}, i\right)$ ) going from $\left(X^{\prime}, Y^{\prime}, i\right)$ to $(X, Y, i)$.

b2). Let $(Z \subset Y \subset X)$ be a stair of closed embeddings. Then it defines coboundary edges $\partial$ from $(Y, Z, i)$ to $(X, Y, i+1)$.

0.5.1. (Co)homological representations of Nori geometric diagrams. If we start not just from the initial category of spaces $\mathcal{C}$, but rather from a pair $(\mathcal{C}, H)$ where $H$ is a cohomology theory, then assuming reasonable properties of this pair, we can define the respective representation $T_{H}$ of $D(\mathcal{C})$ that we will call a (co)homological representation of $D(\mathcal{C})$.

For a survey of such pairs $(\mathcal{C}, H)$ that were studied in the context of Grothendieck's motives, see [HuM-S17], pp. 31-133. The relevant cohomology theories include, in particular, singular cohomology, and algebraic and holomorphic de Rham cohomologies.

Below we will consider the basic example of cohomological representations of Nori diagrams that leads to Nori motives.

0.6. Effective Nori motives ([HuM-S17], pp. 207-208.) Take as a category $\mathcal{C}$, starting object in sec. 2.11 above, the category of varieties $X$ defined over a subfield $k \subset \mathbf{C}$.

We can then define the Nori diagram $D(\mathcal{C})$ as above. This diagram will be denoted Pairs ${ }^{\text {eff }}$ from now on.

The category of effective mixed Nori motives is the diagram category $C\left(\right.$ Pair $\left.s, H^{*}\right)$ where $H^{i}(X, \mathbf{Z})$ is the respective singular cohomology of the analytic space $X^{a n}$ (cf. [HuM-S17], pp. 31-34 and further on).

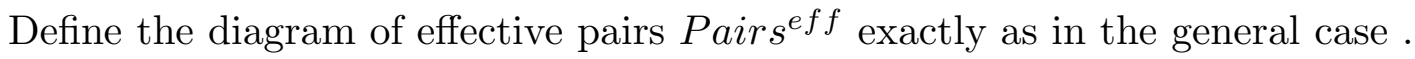

It turns out ([HuM-S17], Proposition 9.1.2. p. 208) that the map

$$
H^{*}: \text { Pairs }^{\text {eff }} \rightarrow \mathbf{Z}-\text { Mod }
$$

sending $(X, Y, i)$ to the relative singular cohomology $H^{i}(X(\mathbf{C}), Y(\mathbf{C}) ; \mathbf{Z})$, naturally extends to a representation of the respective Nori diagram in the category of finitely generated abelian groups $\mathbf{Z}-M o d$. 


\section{NORI GEOMETRIC DIAGRAMS}

We start with a detailed exposition of Nori's construction briefly sketched in 0.5. We extend it by the additional data $(f, \lambda)$ below following D. Arapura's construction of motivic sheaves [Ar13], but tracing his steps in wider categories of topological spaces.

1.1. Definition. The Persistence Diagram $D$ of an appropriate category of topological spaces has vertices of the form $(f: X \rightarrow \mathbf{R}, Y, i, \lambda)$ where

(i) $j: Y \hookrightarrow X$ is a continuous embedding of topological spaces.

(ii) $f: X \rightarrow \mathbf{R}$ is a piecewise continuous map with finitely many "critical values" $t \in \mathbf{R}$. Criticality here means that the homotopy types of $X_{s}=f^{-1}(-\infty, s]$ for $s<t$ and for $s>t$ in a small neighbourhood of $t$ are different.

(iii) $i \in \mathbf{Z}_{+}$is a non-negative integer.

(iv) $\lambda \in \mathbf{R}_{+}$is a non-negative real number.

There are three types of edges in $D$ :

(1) Each continuous map $\phi: X \rightarrow X^{\prime}$ such that the diagrams
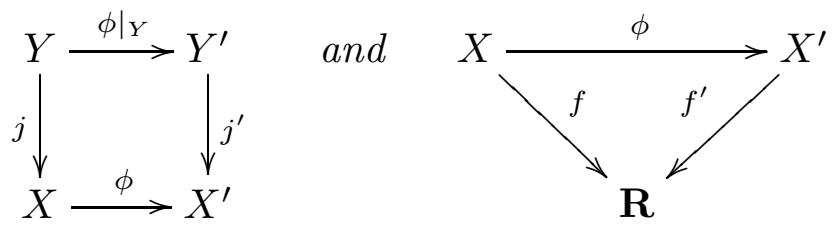

commute, with $\left.\phi\right|_{Y}=\phi \circ j$ the restriction, gives the corresponding edge

$$
\phi_{*}:(f: X \rightarrow \mathbf{R}, Y, i, \lambda) \rightarrow\left(f^{\prime}: X^{\prime} \rightarrow \mathbf{R}, Y^{\prime}, i, \lambda\right) .
$$

(2) Each pair of inclusions $Z \subset Y \subset X$, with compatible maps to $\mathbf{R}$, produces corresponding edge

$$
\partial:(f: X \rightarrow \mathbf{R}, Y, i, \lambda) \rightarrow\left(\left.f\right|_{Y}: Y \rightarrow \mathbf{R}, Z, i-1, \lambda\right) .
$$

(3) For any $\lambda \leq \lambda^{\prime}$, there is an edge

$$
p_{\lambda, \lambda^{\prime}}:(f: X \rightarrow \mathbf{R}, Y, i, \lambda) \rightarrow\left(\ell_{\lambda} \circ f: X \rightarrow \mathbf{R}, Y, i, \lambda^{\prime}\right),
$$

where $\ell_{\lambda}: \mathbf{R} \rightarrow \mathbf{R}$ is the shift map $t \mapsto t-\lambda$. 
This notion of Persistence Diagram of a geometric category is not the same as what is usually called a persistence diagram in the contemporary persistent topology literature (which is a multiset of increasing pairs of numbers in $\mathbf{R}_{+} \cup\{\infty\}$ ). As we will see in Lemma 1.2.1 below, our Persistence Diagrams are closely related to the $(\mathbf{R}, \leq)$-indexed diagrams of $[\mathrm{BuSc} 14]$, hence it is also related to the usual persistence diagrams, as we will explain.

1.2. Linear representations of persistence diagrams. The notion of $(\mathbf{R}, \leq$ )-indexed diagrams in the category of finite dimensional real vector spaces Vec was considered in [BuSco14].

Objects of the category $\operatorname{Vec}^{(\mathbf{R}, \leq)}$ of $[$ BuSco14] are functors $F:(\mathbf{R}, \leq) \rightarrow$ Vec from the thin category $(\mathbf{R}, \leq)$ to the category of finite dimensional real vector spaces. Its morphisms are natural transformations of such functors. It is shown in Section 4 of [BuSco14] that $\operatorname{Vec}^{(\mathbf{R}, \leq)}$ is an abelian category.

Below we will construct a representation $T_{\mathbf{R}}: D \rightarrow \operatorname{Vec}^{(\mathbf{R}, \leq)}$.

Start with the following preliminary notations. First, the inclusions $\iota^{\lambda}: X_{t} \subset$ $X_{t+\lambda}$ of sublevel sets $X_{t}=f^{-1}(-\infty, t]$ and $X_{t+\lambda}=f^{-1}(-\infty, t+\lambda]$ induce maps of the relative homology groups

$$
\iota_{X, i}^{\lambda}: H_{i}\left(X_{t}, Y_{t} ; \mathbf{R}\right) \rightarrow H_{i}\left(X_{t+\lambda}, Y_{t+\lambda} ; \mathbf{R}\right)
$$

where $Y_{t}=\left.f\right|_{Y} ^{-1}(-\infty, t]$ are the induced sublevel sets on $Y$.

Second, an object $V=\left(V_{t}\right)$ of $\operatorname{Vec}^{(\mathbf{R}, \leq)}$ is given by a thin diagram of vector spaces $V=\left(V_{t}\right), t \in \mathbf{R}$.

1.2.1. Lemma-Definition. The following maps define a representation $T_{\mathbf{R}}$ : $D \rightarrow \operatorname{Vec}^{(\mathbf{R}, \leq)}$ of the Persistence Diagram D.

A. On objects:

$$
T_{\mathbf{R}}(f: X \rightarrow \mathbf{R}, Y, i, \lambda)_{t}:=V_{t}:=\operatorname{Range}\left(\iota_{X, i}^{\lambda}\right)_{t} .
$$

B. On edges (using notations from Def. 1.1 above):

$$
T_{\mathbf{R}}\left(\phi_{i}\right)_{t}:=\text { the map Range }\left(\iota_{X, i}^{\lambda}\right)_{t} \rightarrow \operatorname{Range}\left(\iota_{X^{\prime}, i}^{\lambda}\right)_{t} .
$$

Furthermore,

$$
T_{\mathbf{R}}(Z \subset Y \subset X):=\text { the map Range }\left(\iota_{X, i}^{\lambda}\right)_{t} \rightarrow \operatorname{Range}\left(\iota_{X^{\prime}, i}^{\lambda}\right)_{t}
$$


induced by the inclusions of sublevel sets

$$
H_{k}\left(X_{t}, Y_{t} ; \mathbf{R}\right) \rightarrow H_{k}\left(X_{t+\lambda^{\prime}-\lambda}, Y_{t+\lambda^{\prime}-\lambda} ; \mathbf{R}\right)
$$

And finally, for the third type of edges we have morphisms in homology induced by the inclusions of sublevel sets

$$
H_{k}\left(X_{t}, Y_{t} ; \mathbf{R}\right) \rightarrow H_{k}\left(X_{t+\lambda^{\prime}-\lambda}, Y_{t+\lambda^{\prime}-\lambda} ; \mathbf{R}\right)
$$

and the corresponding maps

$$
p_{\lambda, \lambda^{\prime}}: \operatorname{Range}\left(\iota_{X, i}^{\lambda}\right)_{t} \rightarrow \operatorname{Range}\left(\iota_{X, i}^{\lambda^{\prime}}\right)_{t-\lambda}
$$

This Definition, motivated to a large degree by the algebraic-geometric constructions of [Ar13], agrees also with the one in [BuSiSc15], Sec.'2.2.4, where the spaces $\left(V_{t}\right)$ above appear as the persistent homology of $(X, Y)$.

The following remark invokes the main example of persistent homology in the form usually applied to topological data analysis, [Car09]. Recall that, for a finite set of points $P$ embedded in a Euclidean space $\mathbf{R}^{M}$ (or in a more general metric space) the Vietoris-Rips simplicial complex $K(P)_{t}$ at scale $t>0$ has $P$ as 0 -skeleton and has a $k$-simplex for each $(k+1)$-tuple of points $\left\{p_{0}, \ldots, p_{k}\right\} \subset P$ such that $\operatorname{dist}\left(p, p^{\prime}\right) \leq t$ for all pairs $p, p^{\prime} \in\left\{p_{0}, \ldots, p_{k}\right\}$.

1.2.2. Example. Let $P \subset \mathbf{R}^{M}$ be a finite set of points embedded in a Euclidean space (a dataset in some high dimensional ambient space). An $(\mathbf{R}, \leq$ )-diagram of topological spaces (simplicial sets), that is, a functor $P:(\mathbf{R}, \leq) \rightarrow$ Top, is obtained by taking $P(t)$ to be the Vietoris-Rips simplicial complex $K(P)_{t}$ at scale $t \in \mathbf{R}_{+}^{*}$ (and empty for $t<0$ ). In this case the persistent homology as defined above recovers the usual notion of persistent homology of datasets.

1.3. Barcode diagrams. Now we consider the thin indexing category with objects $n \in \mathbf{Z}$ and morphisms $\operatorname{Mor}_{(\mathbf{Z}, \leq)}(n, m)$ consisting of a single morphism for $n \leq m$ and empty othherwise. We pass to the category $\operatorname{Vec}^{(\mathbf{Z}, \leq)}$, with objects that are functors $F:(\mathbf{Z}, \leq) \rightarrow$ Vec from the category $(\mathbf{Z}, \leq)$ to the category of finite dimensional real vector spaces and with morphisms given by natural transformations of these functors. The category $\operatorname{Vec}^{(\mathbf{Z}, \leq)}$ is equivalent to the category of modules over the ring $\mathbf{R}[x]$ (see Lemma 4.5 of [BuSc14]). 
A finite type object in $\operatorname{Vec}^{(\mathbf{R}, \leq)}$ is a functor $F:(\mathbf{R}, \leq) \rightarrow$ Vec such that $F=$ $\oplus_{j=1}^{N} \chi_{\mathcal{I}_{j}}$ where $\chi_{\mathcal{I}_{j}}(t)=\mathbf{R}$ for $t \in \mathcal{I}_{j}$ and zero otherwise and $\chi_{\mathcal{I}_{j}}\left(t \leq t^{\prime}\right)=\mathrm{id} \mathbf{R}$ if $a, b \in \mathcal{I}_{j}$ and zero otherwise (Definition 4.1 of [BuSc14]). By property (ii) of the functions $f: X \rightarrow \mathbf{R}$ in Definition 1.1, the sublevel sets $X_{t}=f^{-1}(-\infty, t]$ have locally constant homotopy type, so in particular the homology $H_{*}\left(X_{t}, Y_{t} ; \mathbf{R}\right)$ is locally constant in $t \in \mathbf{R}$. This implies that the image $T(D)$ in $\operatorname{Vec}^{(\mathbf{R}, \leq)}$ under the representation of Lemma 1.2.1 consists of finite type objects. In particular, by Theorem 4.6 of [BuSc14] finite type objects in $\operatorname{Vec}^{(\mathbf{R}, \leq)}$ are also tame, that is, all but finitely many values $t \in \mathcal{I}$ are regular values, for which there is an open interval $\mathcal{I} \ni t$ such that $V_{t}=F(t)$ is constant on $\mathcal{I}$. The finitely many points $t \in \mathbf{R}$ that are not regular values are called critical points.

The barcode diagram of a finite type object $F$ in $\operatorname{Vec}^{(\mathbf{R}, \leq)}$ is given by the multiset of pairs $\left\{\left(a_{j}, b_{j}\right)\right\}_{j=1}^{N}$ with $a_{j}, b_{j} \in \mathbf{R} \cup\{ \pm \infty\}$ such that $a_{j} \leq b_{j}$ and $\left\{a_{j}, b_{j}\right\}=\partial \mathcal{I}_{j}$ for $F=\oplus_{j} \chi_{\mathcal{I}_{j}}$. The finite $a_{j}, b_{j}$ are also the critical points of the object $F$. Let $-\infty<c_{0}<c_{1}<\cdots<c_{M}<\infty$ denote the ordered sequence of these critical points.

1.3.1. Lemma. The representation $T: D \rightarrow \operatorname{Vec}^{(\mathbf{R}, \leq)}$ of Lemma 1.2.1. determines a representation $T_{\mathbf{Z}}: D \rightarrow \mathrm{Vec}^{(\mathbf{Z}, \leq)}$. Conversely, the datum of $T_{\mathbf{Z}}$ together with the map that assigns to each vertex of $D$ the barcode diagram of its persistent homology completely determine the representation $T: D \rightarrow \operatorname{Vec}^{(\mathbf{R}, \leq)}$.

Proof. Let $F_{\mathbf{Z}}:(\mathbf{Z}, \leq) \rightarrow$ Vec be the functor that assigns to $n \in \mathbf{Z}$ the vector space $F_{\mathbf{Z}}(n)$ given by $F(t)$ for $t \in\left(c_{n}, c_{n+1}\right)$ for $n=0, \ldots, M-1$, the vector space $F(t)$ for $t>c_{M}$ for all $n \geq M$ and the vector space $F(t)$ for $t<c_{0}$ for all $n<0$. To $n \leq m$ the functor $F_{\mathbf{Z}}$ assigns the same morphism $F(n \leq m)$. This determines a finite type object $F_{\mathbf{Z}}$ in the category $\operatorname{Vec}^{(\mathbf{Z}, \leq)}$ associated to the finite type object $F$ in $\operatorname{Vec}^{(\mathbf{R}, \leq)}$. It is clear then that knowing $F_{\mathbf{Z}}$ together with the multiset of points $\left\{\left(a_{j}, b_{j}\right)\right\}_{j=1}^{N}$ (the barcode diagram) uniquely determine $F$.

1.4. Diagram Category. The representation $T_{\mathbf{Z}}: D \rightarrow \operatorname{Vec}^{(\mathbf{Z}, \leq)}$ is a representation of the diagram $D$ in the category of $R$-modules for $R=\mathbf{R}[x]$. Thus, we can apply to this representation the construction of the Nori Diagram Category, see [HuM-S17], Sec. 7.1.2.

Given a representation $T: D \rightarrow R$-Mod of a diagram $D$ into the category of $R$ modules for a commutative ring $R$, the Nori Diagram Category $\mathcal{C}(D, T)$ is defined in the following way (see 0.4 above and Sec. 7.1 .2 of [HuM-S17]). It is the category 
End $(T)$-Mod of modules over the ring of endomorphisms

$$
\begin{gathered}
\operatorname{End}(T)=\left\{\left(\phi_{v}\right)_{v \in V(D)} \mid \phi_{v} \in \operatorname{End}_{R}(T(v)) \text { such that } \phi_{t(e)} \circ T(e)=T(e) \circ \phi_{s(e)},\right. \\
\forall e \in E(D), \text { with source and target } s(e), t(e) \in V(D)\}
\end{gathered}
$$

The category $\mathcal{C}(D, T)$ is an $R$-linear abelian category. We denote by $\Phi: \mathcal{C}(D, T) \rightarrow$ $R$-Mod the forgetful functor.

1.4.1. Lemma. By identifying as above the category $\operatorname{Vec}^{(\mathbf{Z}, \leq)}$ with $\mathbf{R}[x]-M o d$, we obtain the Nori Diagram Category $\mathcal{C}\left(D, T_{\mathbf{Z}}\right)$ associated to the representation $T_{\mathbf{Z}}: D \rightarrow \operatorname{Vec}^{(\mathbf{Z}, \leq)}$ of Lemma 1.3.1, with $\mathcal{C}\left(D, T_{\mathbf{Z}}\right)=\operatorname{End}\left(T_{\mathbf{Z}}\right)-\operatorname{Mod}$.

1.5. Persistent homology on the Nori Diagram Category. We show here that the persistent homology, constructed as in Lemma 1.2.1, determines a faithful exact functor $\mathcal{C}\left(D, T_{\mathbf{Z}}\right) \rightarrow \operatorname{Vec}^{(\mathbf{R}, \leq)}$ on the Nori Diagram Category.

1.5.1. Lemma. Let $\operatorname{Vec}_{f}^{(\mathbf{R}, \leq)}$ denote the full subcategory of $\operatorname{Vec}^{(\mathbf{R}, \leq)}$ with objects that are of finite type. Then

(1) $\operatorname{Vec}_{f}^{(\mathbf{R}, \leq)}$ is an abelian subcategory of $\operatorname{Vec}^{(\mathbf{R}, \leq)}$.

(2) There is an $\mathbf{R}[x]$-linear faithful exact functor $\Psi: \operatorname{Vec}_{f}^{(\mathbf{R}, \leq)} \rightarrow \operatorname{Vec}^{(\mathbf{Z}, \leq)}$ constructed as in Lemma 1.3.1.

Proof. We have to show that $\operatorname{Vec}_{f}^{(\mathbf{R}, \leq)}$ is itself an abelian category and the inclusion functor is exact. This is equivalent to showing that $\operatorname{Vec}_{f}^{(\mathbf{R}, \leq)}$ is closed under taking kernels and cokernels. Let $\alpha: F \rightarrow F^{\prime}$ be a natural transformation of functors $F, F^{\prime}:(\mathbf{R}, \leq) \rightarrow$ Vec that are of the form $F=\oplus_{k=1}^{N} \chi_{\mathcal{I}_{k}}$ and $F^{\prime}=\oplus_{j=1}^{M} \chi_{\mathcal{I}_{j}^{\prime}}$. On objects $t \in \mathbf{R}$ the transformation $\alpha$ acts as an $\mathbf{R}$-linear map $\alpha_{t}: \oplus_{k} \chi_{\mathcal{I}_{k}}(t) \rightarrow$ $\oplus_{j} \chi_{\mathcal{I}_{j}^{\prime}}(t)$. Then there is a finite collection of points $c_{0}<\cdots<c_{m}$ in $\mathbf{R}$, given by the union of the critical points of $F$ and $F^{\prime}$ such that, for all $t \in\left(c_{i}, c_{i+1}\right)$, with $c_{-1}=-\infty$ and $c_{m+1}=+\infty$, the map $\alpha_{t}$ is a linear map $\alpha_{t}: \mathbf{R}^{N_{i}} \rightarrow \mathbf{R}_{M_{i}}$. Since on morphisms the functor $F$ is determined by $\chi_{\mathcal{I}_{k}}\left(t \leq t^{\prime}\right)=$ id if $t, t^{\prime} \in \mathcal{I}_{k}$ and zero otherwise, and similarly for $F^{\prime}$, the natural transformation diagrams

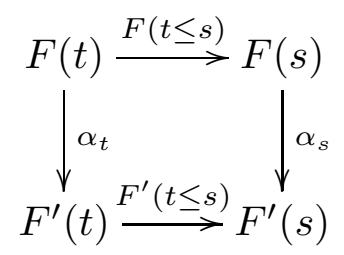


imply that $\alpha_{t}$ is locally constant. Thus, the kernel and cokernel of $\alpha_{t}$ also determine a finite type object in $\operatorname{Vec}_{f}^{(\mathbf{R}, \leq)}$.

Now, consider a functor $F:(\mathbf{R}, \leq) \rightarrow$ Vec which is a finite type object in $\operatorname{Vec}_{f}^{(\mathbf{R}, \leq)}$. Proceeding as above, denote by $r_{0}<\cdots<r_{\ell}$ the critical points of $F$, so that $F(t)$ is locally constant with $F(t)=\mathbf{R}^{N_{i}}$ for all $t \in\left(r_{i}, r_{i+1}\right)$, with $r_{-1}=-\infty$ and $r_{\ell+1}=+\infty$. We can then assign to $F$ a functor $F_{\mathbf{Z}}:(\mathbf{Z}, \leq) \rightarrow$ Vec with $F_{\mathbf{Z}}(n)=F(t)$ with $t \in\left(r_{n}, r_{n+1}\right)$ for $n=0, \ldots, \ell-1$ and $F_{\mathbf{Z}}(n)=F(t)$ with $t<r_{0}$ for all $n<0$ and $F_{\mathbf{Z}}(n)=F(t)$ with $t>r_{\ell}$ for $n \geq \ell$. We also define on morphisms $F_{\mathbf{Z}}(n \leq m)=F\left(t \leq t^{\prime}\right)$ for $t \in\left(r_{n}, r_{n+1}\right)$ (or respectively $t<r_{0}$ or $t>r_{\ell}$ depending on the value of $n$ ) and $t^{\prime} \in\left(r_{m}, r_{m+1}\right)$ (or respectively $t^{\prime}<r_{0}$ or $t^{\prime}>r_{\ell}$ depending on the value of $m$ ). Let $\alpha: F \rightarrow F^{\prime}$ be a natural transformation of functors $F, F^{\prime}:(\mathbf{R}, \leq) \rightarrow$ Vec. We obtain a corresponding natural transformation $\alpha_{\mathbf{Z}}: F_{\mathbf{Z}} \rightarrow F_{\mathbf{Z}}^{\prime}$ by assigning $\alpha_{\mathbf{Z}, n}: F_{\mathbf{Z}}(n) \rightarrow F_{\mathbf{Z}}^{\prime}(n)$ to be the same $\operatorname{map} \alpha_{t}: F(t) \rightarrow F^{\prime}(t)$ for $t \in\left(r_{n}, r_{n+1}\right)$ (or $t<r_{0}$ or $t>r_{\ell}$ depending on $n$ ). The transformation $\alpha_{\mathbf{Z}, n}: F_{\mathbf{Z}}(n) \rightarrow F_{\mathbf{Z}}^{\prime}(n)$ is trivial if and only if $\alpha_{t}: F(t) \rightarrow F^{\prime}(t)$ for $t$ in the corresponding interval is also trivial, so that $\alpha_{\mathbf{Z}}$ is trivial iff $\alpha$ is, hence the functor $F \mapsto F_{\mathbf{Z}}$ is faithful.

Let $\tilde{\mathcal{I}}_{n}$ denote the intervals $\left(-\infty, r_{0}\right)$ for $n<0, \tilde{\mathcal{I}}_{n}=\left(r_{n}, r_{n+1}\right)$ for $n=0, \ldots, \ell-$ 1 and $\left(r_{\ell}, \infty\right)$ for $n \geq \ell$. We have an $\mathbf{R}[x]$-linear structure on $\operatorname{Vec}_{f}^{(\mathbf{R}, \leq)}$ where $x$ acts on $F(t)$ as $F\left(t \leq t^{\prime}\right)$ for $t \in \tilde{\mathcal{I}}_{n}$ and and any $t^{\prime} \in \tilde{\mathcal{I}}_{n+1}$. With respect to this $\mathbf{R}[x]$-linear structure the functor $F \mapsto F_{\mathbf{Z}}$ is $\mathbf{R}[x]$-linear. Moreover, by an argument similar to the one used above to check faithfulness, if we have an exact sequence

$$
0 \longrightarrow F \stackrel{\alpha}{\longrightarrow} F^{\prime} \stackrel{\beta}{\longrightarrow} F^{\prime \prime} \longrightarrow 0
$$

in $\operatorname{Vec}_{f}^{(\mathbf{R}, \leq)}$ we also obtain a corresponding exact sequence

$$
0 \longrightarrow F_{\mathbf{Z}} \stackrel{\alpha_{\mathbf{Z}}}{\longrightarrow} F_{\mathbf{Z}}^{\prime} \stackrel{\beta_{\mathbf{Z}}}{\longrightarrow} F_{\mathbf{Z}}^{\prime \prime} \longrightarrow 0
$$

Hence the functor $\Psi$ mapping $F \mapsto F_{\mathbf{Z}}$ and $\alpha \mapsto \alpha_{\mathbf{Z}}$ is a faithful exact $\mathbf{R}[x]$-linear functor $\operatorname{Vec}_{f}^{(\mathbf{R}, \leq)} \rightarrow \operatorname{Vec}^{(\mathbf{Z}, \leq)}$.

\subsubsection{Proposition. Persistent homology determines a faithful exact functor}

$$
P H_{*}: \mathcal{C}\left(D, T_{\mathbf{Z}}\right) \rightarrow \operatorname{Vec}_{f}^{(\mathbf{R}, \leq)} .
$$


Proof. The Nori Diagram Category $\mathcal{C}(D, T)$ of a representation $T: D \rightarrow R-\mathrm{Mod}$ of a diagram $D$ satisfies the following universal property: given any $R$-linear abelian category $\mathcal{A}$, a representation $T_{\mathcal{A}}: D \rightarrow \mathcal{A}$, and an $R$-linear faithful exact functor $\Psi: \mathcal{A} \rightarrow R$-Mod such that $\Psi \circ T_{\mathcal{A}}=T$, then there exists a faithful exact functor $\Phi_{\mathcal{A}}: \mathcal{C}(D, T) \rightarrow \mathcal{A}$ such that the following diagram commutes:

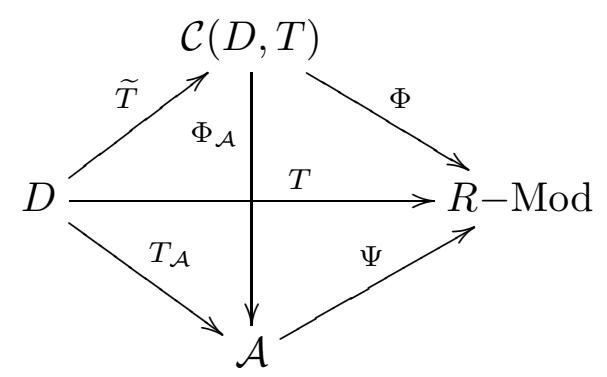

As we mentioned in Sec. 0.4 above, the category $\mathcal{C}(D, T)$ is in fact completely characterized by this property up to unique equivalence of categories. (see Sec. 7.1.3 of [HuM-S17], Sec. 7.1.3.)

Now, apply this universal property of the Nori Diagram Category to the following case: $D$ is the Persistence Diagram of Definition $1.1 ; T_{\mathbf{Z}}: D \rightarrow \mathbf{R}[x]-\operatorname{Mod}$ is the representation of Lemma $1.5 .1 ; \mathcal{A}=\operatorname{Vec}_{f}^{(\mathbf{R}, \leq)}$, with the representation $T$ : $D \rightarrow \operatorname{Vec}_{f}^{(\mathbf{R}, \leq)}$ of Lemma 1.2.1, and the functor $\Psi: \operatorname{Vec}_{f}^{(\mathbf{R}, \leq)} \rightarrow \mathbf{R}[x]-\operatorname{Mod}$ is the one of Lemma 1.5.1. To this purpose it suffices to check that the functors $\Psi, T, T_{\mathbf{Z}}$ satisfy the composition property $\Psi \circ T=T_{\mathbf{Z}}$, which is true by construction (compare Lemma 1.5.1 with Lemma 1.4.1). We then obtain a faithful exact functor $P H_{*}: \mathcal{C}\left(D, T_{\mathbf{Z}}\right) \rightarrow \operatorname{Vec}_{f}^{(\mathbf{R}, \leq)}$ that completes the commutative diagram

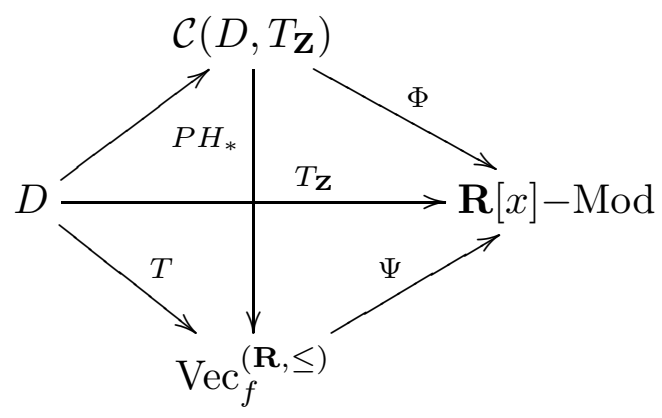


1.6. The product structure. We will show here that the Persistence Diagram $D$ of Definition 1.1 has the structure of a graded diagram with a commutative product with unit, in the sense of Definition 8.1.3 of [HuM-S17]. Recall from this definition that a graded diagram $D$ is a diagram endowed with a map deg: $V(D) \rightarrow$ $\mathbf{Z} / 2 \mathbf{Z}$ extended to $\operatorname{deg}: E(D) \rightarrow \mathbf{Z} / 2 \mathbf{Z}$ by $\operatorname{deg}(e)=\operatorname{deg}(s(e))-\operatorname{deg}(t(e))$. The product $D \times D$ is the diagram with vertices the pairs $(v, w) \in V(D) \times V\left(D^{\prime}\right)$ and edges of the form $(e, i d)$ or $\left(i d, e^{\prime}\right)$. A product structure on $D$ is a map of graded diagrams (a degree preserving map of directed graphs) $D \times D \rightarrow D$ together with a choice of edges

$$
\begin{gathered}
\alpha_{v, w}: v \times w \rightarrow w \times v, \quad \forall v, w \in V(D) \\
\beta_{v, w, u}: v \times(w \times u) \rightarrow(v \times w) \times u, \\
\beta_{v, w, u}^{\prime}:(v \times w) \times u \rightarrow v \times(w \times u), .
\end{gathered}
$$

for all $v, w, u \in V(D)$. A unit is a vertex $\mathbf{1}$ with $\operatorname{deg}(\mathbf{1})=0$ and edges $u_{v}: v \rightarrow \mathbf{1} \times v$ for all $v \in V(D)$.

1.6.1. Lemma. The Persistence Diagram D of Definition 1.1 is a graded diagram with commutative product and unit.

Proof. Define the $\mathbf{Z}_{2}$-grading by $\operatorname{deg}(f: X \rightarrow \mathbf{R}, Y, k, \lambda)=k \bmod 2$. The product $D \times D \rightarrow D$ is given by

$$
\begin{gathered}
(f: X \rightarrow \mathbf{R}, Y, k, \lambda) \times\left(f^{\prime}: X^{\prime} \rightarrow \mathbf{R}, Y^{\prime}, k^{\prime}, \lambda^{\prime}\right):= \\
\left(X \times_{\mathbf{R}} X^{\prime} \rightarrow \mathbf{R}, X \times_{\mathbf{R}} Y^{\prime} \cup Y \times_{\mathbf{R}} X^{\prime}, k+k^{\prime}, \lambda+\lambda^{\prime}\right),
\end{gathered}
$$

where $X \times_{\mathbf{R}} X^{\prime}$ is the fibered product:

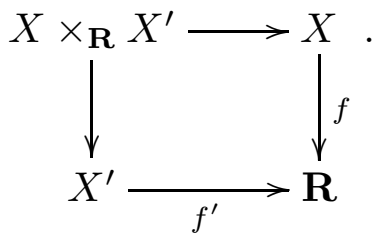

This product satisfies the identities

$$
\left(X \times_{\mathbf{R}} X^{\prime}\right)_{t}=\left\{\left(x, x^{\prime}\right) \in X \times X^{\prime}: f(x)=f^{\prime}\left(x^{\prime}\right) \leq t\right\}=X_{t} \times_{\mathbf{R}} X_{t}^{\prime} .
$$


The unit vertex is given by (id $: \mathbf{R} \rightarrow \mathbf{R}, \emptyset, 0,0)$. The edges $\alpha_{v, w}, \beta_{v, w, u}$ and $\beta_{v, w, u}^{\prime}$ are the natural homeomorphisms of topological spaces compatible with the maps. This finishes the proof.

We recall now that in the situation of Lemma 1.6.1 one can define a subclass of representations of $D$ that are compatible with grading and commutative product. Namely, according to the Definition 8.1.3 of [HuM-S17], the compatibility conditions for a representation $T \rightarrow R$-Proj of a graded diagram $D$ are given by the existence of isomorphisms

$$
\tau_{v, w}: T(v \times w) \stackrel{\simeq}{\longrightarrow} T(v) \otimes T(w)
$$

for all $v, w \in V(D)$, with the following properties:

$$
T(v) \otimes T(w) \stackrel{\tau_{v, w}^{-1}}{\longrightarrow} T(v \times w) \stackrel{T\left(\alpha_{v, w}\right)}{\longrightarrow} T(w \times v) \stackrel{\tau_{w, v}}{\longrightarrow} T(w) \otimes T(v)
$$

is equal to multiplication by $(-1)^{\operatorname{deg}(v) \operatorname{deg}(w)}$; the $\beta$-maps satisfy $T\left(\beta_{v, w, u}\right)^{-1}=$ $T\left(\beta_{v, w, u}^{\prime}\right)$, and moreover

$$
\begin{aligned}
& \tau_{v, w^{\prime}} \circ T(1, e)=(\mathrm{id} \otimes T(e)) \circ \tau_{v, w}: T(v \times w) \rightarrow T(v) \otimes T\left(w^{\prime}\right) \\
& \tau_{v^{\prime}, w} \circ T(e, 1)=(T(e) \otimes \mathrm{id}) \circ \tau_{v, w}: T(v \times w) \rightarrow T\left(v^{\prime}\right) \otimes T(w) \\
& T(v \times(w \times u)) \stackrel{T\left(\beta_{v, w, u}\right)}{\longrightarrow} T((v \times w) \times u) \\
& \downarrow \tau \circ \tau \quad \downarrow \tau \circ \tau \\
& T(v) \otimes(T(w) \otimes T(u)) \stackrel{\simeq}{\longrightarrow}(T(v) \otimes T(w)) \otimes T(u))
\end{aligned}
$$

and similarly for the inverse $T\left(\beta_{v, w, u}^{\prime}\right)$.

1.7. Good persistence vertices. In order to define a tensor structure on the Nori Diagram Category $\mathcal{C}\left(D, T_{\mathbf{Z}}\right)$ of the Persistence Diagram we need to proceed in a way similar to that adopted in the construction of the category of Nori motives, see [HuM-S17], Sec. 9. Indeed, because of the Künneth product formula

$$
H_{k}\left(\left(X \times_{\mathbf{R}} X^{\prime}\right)_{t},\left(X \times_{\mathbf{R}} Y^{\prime} \cup Y \times_{\mathbf{R}} X^{\prime}\right)_{t} ; \mathbf{R}\right) \simeq \oplus_{i+j=k} H_{i}\left(X_{t}, Y_{t} ; \mathbf{R}\right) \otimes H_{j}\left(X_{t}^{\prime}, Y_{t}^{\prime} ; \mathbf{R}\right)
$$


where $\left(X \times_{\mathbf{R}} X^{\prime}\right)_{t}=X_{t} \times_{\mathbf{R}} X_{t}^{\prime}$ and $\left(X \times_{\mathbf{R}} Y^{\prime} \cup Y \times_{\mathbf{R}} X^{\prime}\right)_{t}=X_{t} \times_{\mathbf{R}} Y_{t}^{\prime} \cup Y_{t} \times_{\mathbf{R}} X_{t}^{\prime}$, this relative homology is compatible with the product structure on $D$ in the case where these homology groups are supported in a single degree. As in the case of Nori motives, we can introduce a class of "good objects" for which the persistent homology is concentrated in a single degree.

1.7.1. Definition. A vertex $(f: X \rightarrow \mathbf{R}, Y, k, \lambda)$ of the Persistence Diagram $D$ is a "good persistence vertex" if the persistent homology

$$
H P_{j}(f: X \rightarrow \mathbf{R}, Y, k, \lambda)_{t}=\operatorname{Range}\left(H_{j}\left(X_{t}, Y_{t} ; \mathbf{R}\right) \rightarrow H_{j}\left(X_{t+\lambda}, Y_{t+\lambda} ; \mathbf{R}\right)\right)
$$

satisfies $H P_{j}(f: X \rightarrow \mathbf{R}, Y, k, \lambda)_{t}=0$ for all $j \neq k$.

Simple examples of good persistence vertices can be constructed as follows. Let $X$ be a smooth $n$-dimensional compact manifold and let $f: X \rightarrow \mathbf{R}$ be a Morse function, which has finitely many critical points $x_{1}, \ldots, x_{m}$ in $X$ with critical values $c_{1}<\cdots<c_{m}$ in $\mathbf{R}$. The sublevel sets $X_{t}=f^{-1}(-\infty, t]$ have homotopy type that remains constant when $t$ varies in each of the intervals $\left(-\infty, c_{1}\right),\left(c_{k}, c_{k+1}\right)$ with $k=1, \ldots, m-1$, and $\left(c_{m}, \infty\right)$ and changes across the critical values by a handle attachment. Let $B$ be an open $n$-ball in $X$ that does not contain any critical point and such that the sublevel sets $B_{t}=\left.f\right|_{B} ^{-1}(-\infty, t]$ are either empty or a contractible set that is open in the induced topology on $X_{t}$, or all of $B$. Let $Y=X \backslash B$. Then the relative homology $H_{k}\left(X_{t}, Y_{t} ; \mathbf{R}\right)=H_{k}\left(X_{t}, X_{t} \backslash B_{t} ; \mathbf{R}\right)$ is a local homology and is trivial for $k \neq 0$ and is either trivial or a single copy of $\mathbf{R}$ for $k=n$. This gives an example where the persistent homology is concentrated in degree $k=n$.

Indeed, if we assume all the topological spaces involved are CW complexes, it is always possible to compute the homology via a cellular filtration by good spaces. Indeed we have the following result (Theorem 2.35 of [Hat02]).

1.7.2. Lemma. Given a $C W$ complex $X$ with skeleta $X^{(n)}$, the homology $H_{k}(X ; \mathbf{Z})$ is computed as the homology of a complex

$$
\cdots \rightarrow H_{j}\left(X^{(j)}, X^{(j-1)} ; \mathbf{Z}\right) \rightarrow H_{j}\left(X^{(j-1)}, X^{(j-2)} ; \mathbf{Z}\right) \rightarrow \cdots
$$

with the maps given by the boundary maps of the pair of inclusions $X^{(j-2)} \subset$ $X^{(j-1)} \subset X^{(j)}$. The relative homology $H_{n}\left(X^{(j)}, X^{(j-1)} ; \mathbf{Z}\right)$ is trivial for $n \neq j$ and a free abelian group for $n=j$ spanned by the $j$-cells of $X$.

1.7.3. Lemma. Under the assumption that all spaces considered are cellular with cellular maps, the representations $T: D \rightarrow \operatorname{Vec}^{(\mathbf{R}, \leq)}$ and $T_{\mathbf{Z}}: D \rightarrow \operatorname{Vec}_{f}^{(\mathbf{Z}, \leq)}$ of Lemma 1.2.1 and Lemma 1.3.1 are unital graded multiplicative representations. 
Proof. First notice that the image of $T_{\mathbf{Z}}: D \rightarrow \operatorname{Vec}^{(\mathbf{Z}, \leq)}=\mathbf{R}[x]$-Mod lies in the subcategory $\operatorname{Vec}_{f}^{(\mathbf{Z}, \leq)}$ of finite type. This is in fact a subcategory of the category $\mathbf{R}[x]-$ Proj of finite projective module over $\mathbf{R}[x]$. Then observe that, under the cellular assumption, Lemma 1.7.2 implies that, if we consider the Nori Diagram Category built on a subdiagram of the Persistence Diagram where all the vertices are good persistence vertices, the resulting $C\left(D^{\text {good }}, T_{\mathbf{Z}}\right)$ contains all the objects $\left(X^{(j)}, X^{(j-1)}, j, \lambda\right)$ and $\left(Y^{(j)}, Y^{(j-1)}, j, \lambda\right)$ for every vertex $(f: X \rightarrow \mathbf{R}, Y, j, \lambda)$ of the Persistence Diagram. Thus, there is an object in the Nori Diagram Category $C\left(D^{\text {good }}, T_{\mathbf{Z}}\right)$ whose image under the forgetful functor to $\operatorname{Vec}^{(\mathbf{Z}, \leq)}$ is the same as the image $T_{\mathbf{Z}}(f: X \rightarrow \mathbf{R}, Y, j, \lambda)$. This implies that we can equivalently use the categories $C\left(D, T_{\mathbf{Z}}\right)$ and $C\left(D^{\text {good }}, T_{\mathbf{Z}}\right)$. Using the latter, we can define the product structure, finishing the proof.

Notice that essentially the same argument was used in [HuM-S17], Sec. 9 in order to construct the product structure on Nori effective motives. The argument is simplified here because we work in a topological setting, hence we can directly use cellular homology as in Lemma 1.7.2, instead of having to use Beilinson's fundamental lemma for the cohomology of affine varieties and complexes of varieties to pass from affine to more general varieties.

The Tannakian formalism. An advantage of reformulating the categorical construction of persistent homology of [BuSco14], [BuSiSco15], [BluLes17] in terms of the formalism of Nori diagrams and Nori motives, as we did in the previous subsections, is the fact that this formulation comes endowed with natural symmetries associated to persistent homology which are not immediately visible otherwise, namely the associated Tannakian formalism.

In the category of Nori motives, one passes from effective motives to the localization with respect to $\left(\mathbf{G}_{m},\{1\}, 1\right)$ (inverting the Lefschetz motive) to obtain a rigid abelian tensor category to which the Tannakian formalism can be applied. In our setting we work with weaker properties, as we will discuss more in Section 4 where we present a more general formalism based on Nori diagram for persistent phenomena. We do not assume that the category we construct is a rigid tensor category, although we will assume that it has a tensor structure, obtained via approximations using filtrations by good objects as explained above. Thus, instead of the group scheme that one expects to obtain as Tannakian Galois group in the case of rigid tensor categories, we only have a monoid scheme, obtained as follows.

1.8.1. Proposition. The representation $T_{\mathbf{Z}}: D \rightarrow \operatorname{Vec}_{f}^{(\mathbf{Z}, \leq)}$ induces an equiva- 
lence between the Nori Diagram Category $\mathcal{C}\left(D, T_{\mathbf{Z}}\right)$ and the category of finitely generated comodules over a bialgebra $\mathcal{A}\left(D, T_{\mathbf{Z}}\right)$, which defines a pro-algebraic monoid scheme $\operatorname{Spec}\left(\mathcal{A}\left(D, T_{\mathbf{Z}}\right)\right)$.

Proof. As before, we view the representation $T_{\mathbf{Z}}: D \rightarrow \operatorname{Vec}_{f}^{(\mathbf{Z}, \leq)}$ as taking values in the category $\mathbf{R}[x]$-Proj of finitely generated projective modules over the Dedekind domain $\mathbf{R}[x]$. We can then apply Theorem 7.1.12 of [HuM-S17] and we obtain that the Nori Diagram Category $\mathcal{C}\left(D, T_{\mathbf{Z}}\right)$ is equivalent to the category of finitely generated comodules over the coalgebra $\mathcal{A}\left(D, T_{\mathbf{Z}}\right)$ given by the colimit

$$
\mathcal{A}\left(D, T_{\mathbf{Z}}\right)=\operatorname{colim}_{D_{F}} \operatorname{End}\left(\left.T_{\mathbf{Z}}\right|_{D_{F}}\right)^{\vee}
$$

over finite sub-diagrams $D_{F}$ with $\vee$ the $\mathbf{R}[x]$-dual.

Indeed, as shown in Sec. 7.5.1 of [HuM-S17], if $R$ is a Dedeking domain, then for the $R$-algebra $E=\operatorname{End}\left(\left.T\right|_{D_{F}}\right)$ with $D_{F}$ a finite diagram and $T$ a representation of a Nori diagram $D$, the $R$-dual $E^{\vee}=\operatorname{Hom}_{R}(E, R)$ has the property that the canonical $\operatorname{map} E^{\vee} \otimes_{R} E^{\vee} \rightarrow \operatorname{Hom}\left(E, E^{\vee}\right) \simeq\left(E \otimes_{R} E\right)^{\vee}$ is an isomorphism. Thus, an $E$ module that is finitely generated projective as an $R$-module carries the structure of an $E^{\vee}$-comodule. The coalgebra $\mathcal{A}\left(D, T_{\mathbf{Z}}\right)$ also carries an algebra structure induced by the monoidal structure of $\mathcal{C}\left(D, T_{\mathbf{Z}}\right)$, see Sections 7.1.4 and 8.1 of [HuMS17]. Thus, $\mathcal{A}\left(D, T_{\mathbf{Z}}\right)$ determines a pro-algebraic monoid $\operatorname{scheme} \operatorname{Spec}\left(\mathcal{A}\left(D, T_{\mathbf{Z}}\right)\right)$ (see Section 7.1.4 of [HuM-S17]). This completes the proof.

In the more general setting of Section 4 below we will only assume that the target category of our fiber functors is an abelian tensor category, but not necessarily a category $R$-Proj with $R$ a Dedekind domain as here above, with the Tannakian formalism of Sections 7.1.2-7.1.4 of [HuM-S17]. Indeed, the target category in general will be a category $\operatorname{Vec}^{(S, \leq)}$ for some poset $(S, \leq)$. This has a tensor structure obtained by identifying it with the category of covariant functors $\mathcal{F}((S, \leq)$, Vec $)$, endowed with the pointwise monoidal structure induced by the monoidal structure on Vec.

\section{THIN CATEGORIES AND PERSISTENCE}

In this section, we describe a slightly more general framework for persistence constructions. It was sketched in the Introduction and its various more precise versions will be given in the remaining Sections of the article.

We start with a category of geometric objects, and an indexing system for persistence which is given by a thin category. 
2.1. Geometric poset objects and thin categories. Let $\mathcal{C}_{\text {geom }}$ be a category of geometric objects (topological spaces, simplicial sets, smooth manifolds, algebraic varieties, etc.). Whenever it is fixed, we refer to its morphisms as "geometric morphisms" etc.

2.1.1. Definition. A poset object in $\mathcal{C}_{\text {geom }}$ is an object $S$ together with a subobject $\mathcal{R} \subset S \times S$ with the following properties:

- $(s, s) \in \mathcal{R}$ for all $s \in S$,

- If $\left(s, s^{\prime}\right) \in \mathcal{R}$ and $\left(s^{\prime}, s^{\prime \prime}\right) \in \mathcal{R}$, then $\left(s, s^{\prime \prime}\right) \in \mathcal{R}$;

- If $\left(s, s^{\prime}\right) \in \mathcal{R}$ and $\left(s^{\prime}, s\right) \in \mathcal{R}$, then $s=s^{\prime}$.

The relation $\left(s, s^{\prime}\right) \in \mathcal{R}$ we also denote by $s \leq s^{\prime}$.

As was explained in Sec. 0.2 above, the notions of a poset and of a thin category essentially coincide.

2.1.2. Remark. The assumption that $\mathcal{C}_{\text {geom }}$ is a category of geometric objects implies that points and subobjects are defined in the usual geometric terms. In a more general setting, one needs to use a formulation that depends on a categorical notion of points in terms of the functor of points. We will discuss this in the next section.

2.2. Persistence modules. It is a general fact that the category of covariant functors $F: \mathcal{B} \rightarrow \mathcal{A}$ from a small category $\mathcal{B}$ to an abelian category $\mathcal{A}$ is itself abelian (see for instance Proposition 44 of [Murf06]). Thus, we can give the following definition.

2.2.1. Definition. Given a poset $(S, \leq)$ and an abelian category $\mathcal{A}$, let $\mathcal{A}^{(S, \leq)}$ be the abelian category whose objects are the covariant functors $F:(S, \leq) \rightarrow \mathcal{A}$ and morphisms the natural transformations of such functors. Objects of $\mathcal{A}^{(S, \leq)}$ will be referred to as the $(S, \leq)$-persistence modules in $\mathcal{A}$. In the case where $\mathcal{A}=R$-Mod, we refer to objects in $R$-Mod ${ }^{(S, \leq)}$ as $(S, \leq)$-persistence $R$-modules.

2.3. Sublevel objects. As above let $\mathcal{C}_{\text {geom }}$ be a category of geometric objects and let $(S, \leq)$ be a poset object in $\mathcal{C}_{\text {geom }}$ as in Definition 2.1.1.

2.3.1. Definition. Consider a pair $(X, f: X \rightarrow S)$ where $X$ is an geometric object and $f$ its morphism to the poset object $(S, \leq)$. For any $s \in S$ we define the sublevel objects $X_{f, s} \subset X$ as $X_{f, s}:=\{x \in X: f(x) \leq s \in S\}$. They define inclusion maps $j_{X, s, s^{\prime}}: X_{f, s} \hookrightarrow X_{f, s^{\prime}}$ for all $s \leq s^{\prime}$ in $S$. 
2.4. Persistent functors. Consider now a geometric object which is a poset $(S, \leq)$ in its category, and let $\mathcal{H}: \mathcal{C}_{\text {geom }} \rightarrow \mathcal{A}$ be a functor with values in an abelian category $\mathcal{A}$.

Denote by $\tilde{\mathcal{C}}_{\text {geom }}^{S}$ the category of "geometric families over a base $S$ ". More precisely, one of its object is a pair consisting of a geometric object and geometric morphism $(X, f: X \rightarrow S)$. A morphism $(X, f) \rightarrow\left(X^{\prime}, f^{\prime}\right)$ is a geometric morphism $\varphi: X \rightarrow X^{\prime}$ such that $f^{\prime} \circ \varphi=f$.

Any functor $\mathcal{H}: \mathcal{C}_{\text {geom }} \rightarrow \mathcal{A}$ as above determines the functor

$$
\tilde{\mathcal{H}}: \tilde{\mathcal{C}}_{\text {geom }}^{S} \rightarrow \mathcal{A}^{(S, \leq)}
$$

as follows. It sends each family $(X, f)$ to the object

$$
\tilde{\mathcal{H}}(X, f: X \rightarrow S):=F_{X, f, S}: s \mapsto \mathcal{H}\left(X_{f, s}\right)
$$

and each morphism $\varphi: X \rightarrow X^{\prime}$ of families to the natural transformation of the functors $F_{X, f, S} \rightarrow F_{X^{\prime}, f^{\prime}, S}$ given by

$$
\tilde{\mathcal{H}}(\varphi):=\mathcal{H}\left(\varphi_{s}\right): \mathcal{H}\left(X_{f, s}\right) \rightarrow \mathcal{H}\left(X_{f^{\prime}, s}^{\prime}\right) .
$$

Here $\varphi_{s}: X_{f, s} \rightarrow X_{f^{\prime}, s}^{\prime}$ is the restriction $\varphi_{s}=\left.\varphi\right|_{X_{f, s}}$ which maps to $X_{f^{\prime}, s}^{\prime}$ because of the compatibility $f^{\prime} \circ \varphi=f$.

2.4.1. Definition. For $\lambda \in S$, the persistence functor $P \mathcal{H}_{\lambda}: \tilde{\mathcal{C}}_{\text {geom }} \rightarrow \mathcal{A}^{(S, \leq)}$ of the functor $\mathcal{H}: \mathcal{C}_{\text {geom }} \rightarrow \mathcal{A}$ is defined as follows.

It sends each family $f: X \rightarrow S$ ) to

$$
P \mathcal{H}_{\lambda}(X, f: X \rightarrow S):=s \mapsto \operatorname{Range}\left(\mathcal{H}\left(j_{X_{s, \lambda}}\right)\right)
$$

for $s \leq \lambda$ and zero otherwise, where

$$
j_{X_{s, \lambda}}: X_{f, s} \hookrightarrow X_{f, \lambda}, \quad \text { for } s \leq \lambda
$$

and the induced morphism in $\mathcal{A}$

$$
\mathcal{H}\left(j_{X_{s, \lambda}}\right): \mathcal{H}\left(X_{f, s}\right) \rightarrow \mathcal{H}\left(X_{f, \lambda}\right)
$$


On morphisms $\varphi: X \rightarrow X^{\prime}$ with $f^{\prime} \circ \varphi=f$ it is defined as the restriction of $\tilde{\mathcal{H}}(\varphi)$ to Range $\left(\mathcal{H}\left(j_{X_{s, \lambda}}\right)\right)$ which takes values in $\operatorname{Range}\left(\mathcal{H}\left(j_{X_{s, \lambda}^{\prime}}\right)\right)$.

2.5. Example: persistent topology of graphs. Let $H$ be a finite directed graph of a thin category. Consider families of finite directed graphs $(G, f: G \rightarrow H)$ over $H$. Let $G_{f, v}$ with $v \in V(H)$ be the respective sublevel graphs. The graph $G_{f, v}$ is the induced subgraph of $G$ on the set of vertices $w \in V(G)$ such that there is a path of directed edges in $H$ between $f(w)$ and $v$. The $(H, \leq)$-persistent topology of $G$ is then specified by the persistent connected components

$$
\text { Range }\left(H_{0}\left(G_{f, v} ; \mathbf{Z}\right) \rightarrow H_{0}\left(G_{f, v^{\prime}} ; \mathbf{Z}\right)\right) \text { for } v \leq v^{\prime} \in V(H),
$$

and the persistent cycles

$$
\text { Range }\left(H_{1}\left(G_{f, v} ; \mathbf{Z}\right) \rightarrow H_{1}\left(G_{f, v^{\prime}} ; \mathbf{Z}\right)\right) \text { for } v \leq v^{\prime} \in V(H) .
$$

2.6. Example: persistent Orlik-Solomon algebras. Let $A$ be a hyperplane arrangement. Denote by $L(A)$ the associated intersection poset, ordered by reverse inclusion. We consider morphisms $\varphi$ of hyperplane arrangements given by linear maps of the ambient space that map one arrangement to the other and we write $L(\varphi)$ for the induced map of intersection posets. We fix one arrangement $A$ and we consider pairs $(B, \varphi)$ of arrangements endowed with a morphism $\varphi$ to $A$. The intersection poset $L(A)$ determines a structure of poset of topological spaces (equivalently, an object in $\operatorname{Top}^{(L(A), \leq)}$ in the notation of [BuSc14], [BuSiSc15]) on the hyperplane arrangement complement $M(A)$, with inclusions $M(A)_{s} \hookrightarrow M(A)_{s^{\prime}}$ for $s \leq s^{\prime}$ in $L(A)$. Given a morphism of arrangements $\varphi: B \rightarrow A$, defined as above, we obtain similarly a structure of poset of topological spaces on the complement $M(B)$ indexed by the poset $L(A)$. The families $M(B)_{s}$ with $s \in L(A)$ with the inclusions $M(B)_{s} \hookrightarrow M(B)_{s^{\prime}}$ for $s \leq s^{\prime}$ in $L(A)$ form the system of sublevel objects described above. The cohomology $H^{*}(M(B)$ ) (with coefficients in a field $\mathbf{K})$ of a hyperplane arrangement complement is the Orlik-Solomon algebra $O S(B)=H^{*}(M(B))$. We consider the cohomology $H^{*}\left(M(B)_{s}\right)$ with the maps induced by the inclusions $M(A)_{s} \hookrightarrow M(A)_{s^{\prime}}$. In order to have a covariant functor, we can consider the homology $O S^{\vee}(B):=H_{*}(M(B))$ with its structure of module over the exterior algebra on $H^{1}(M(B))$, [EPY03]. The associated persistent functor is given by

$$
P O S_{\lambda}^{\vee}(B):=\text { Range }\left(H_{*}\left(M(B)_{s}\right) \rightarrow H_{*}\left(M(B)_{\lambda}\right)\right), \text { for } s \leq \lambda \in L(A) .
$$


Notice that we could also consider the persistent homology of a hyperplane arrangement complement $M(B)$ with the persistence indexed by its own intersection poset $L(B)$. The case considered above, where one considers arrangements $B$ endowed with maps to a fixed arrangement $A$ and persistence with respect to the fixed $L(A)$ provides a uniform choice of the poset indexing the persistence modules. Allowing the indexing poset to depend on the arrangement, as in the case where one uses $L(B)$ has advantages too, for example because there are in general few linear maps of the ambient space that induce maps between two given arrangements.

2.7. Example: persistent Tate motives. Beilinson, Goncharov, Schechtman and Varchenko in [BGSV90] conjectured that, over a number field $\mathbf{K}$ the category of mixed Tate motive is generated by motives of the form

$$
\mathbf{m}\left(\mathbf{P}^{n} \backslash A, A \backslash(A \cap B)\right)
$$

where $A$ and $B$ are hyperplane arrangements in general position. These are the motives whose cohomological realization gives the middle dimensional relative cohomology of the pair $\left(\mathbf{P}^{n} \backslash A, A \backslash(A \cap B)\right)$. We can consider a setting as in the previous subsection, where one covers the hyperplane arrangement complement with sublevel sets indexed by a poset and correspondingly consider motives of the form $\mathbf{m}\left(\left(\mathbf{P}^{n} \backslash A\right)_{s},(A \backslash(A \cap B))_{s}\right)$ and persistent objects

Range $\left(\mathbf{m}\left(\left(\mathbf{P}^{n} \backslash A\right)_{s},(A \backslash(A \cap B))_{s}\right) \rightarrow \mathbf{m}\left(\left(\mathbf{P}^{n} \backslash A\right)_{s^{\prime}},(A \backslash(A \cap B))_{s^{\prime}}\right)\right)$ for $s \leq s^{\prime}$.

\section{SUBLEVEL SIEVES AND PERSISTENCE}

In the setting described above we have assumed that we work with a category $\mathcal{C}_{\text {geom }}$ of geometric objects and we have used the geometric notion of points to define sublevel sets and persistence. We consider here more general categories $\mathcal{C}$ for which objects do not necessarily have points in the geometric sense. However, they always have a "functor of points" in Grothendieck's sense: for an object $X$ in $\mathcal{C}$ and another object $A$, an $A$-point of $X$ is a morphism $\varphi: A \rightarrow X$ in $\operatorname{Mor}_{\mathcal{C}}(A, X)$. We will use here this approach to define a notion of persistent functors $P \mathcal{H}$ associated to certain functors $\mathcal{H}: \mathcal{C} \rightarrow \mathcal{A}$ with values in an abelian category.

3.1. Functor of points and Poset functors. Let $\mathcal{C}$ be a category and $X \in \operatorname{Obj}(\mathcal{C})$. The functor of points $\pi_{X}: \mathcal{C} \rightarrow$ Sets is a contravariant functor with 
$\pi_{X}(A)=\operatorname{Mor}_{\mathcal{C}}(A, X)$ and $\pi_{X}(\varphi: B \rightarrow A)=-\circ \varphi: \operatorname{Mor}_{\mathcal{C}}(A, X) \rightarrow \operatorname{Mor}_{\mathcal{C}}(B, X)$. The object $X$ is completely determined by its functor of points $\pi_{X}$ in the sense that a natural tranformation $\eta: \pi_{X} \rightarrow \pi_{Y}$ determines a morphism $f: X \rightarrow Y$ in such a way that natural equivalences are in $(1,1)$-correspondence with isomorphisms of the respective objects.

3.1.1. Definition. Let $\mathcal{C}$ be a category and $S$ its object. Let $\pi_{S}: \mathcal{C} \rightarrow$ Sets be the functor of points of $S$. A poset functor on $S$ is a contravariant functor $\mathcal{R}_{(S, \leq)}$ : $\mathcal{C} \rightarrow$ Sets given on objects by the assignment of a subset $\mathcal{R}_{(S, \leq)}(A) \subseteq \pi_{S}(A) \times \pi_{S}(A)$ with the following properties:

(1) $\left(p_{A}, p_{A}\right) \in \mathcal{R}_{(S, \leq)}(A)$ for all $p_{A} \in \pi_{S}(A)=\operatorname{Mor}_{\mathcal{C}}(A, S)$;

(2) $\left(p_{A}, p_{A}^{\prime}\right) \in \mathcal{R}_{(S, \leq)}(A)$ and $\left(p_{A}^{\prime}, p_{A}^{\prime \prime}\right) \in \mathcal{R}_{(S, \leq)}(A)$ implies $\left(p_{A}, p_{A}^{\prime \prime}\right) \in \mathcal{R}_{(S, \leq)}(A)$;

(3) $\left(p_{A}, p_{A}^{\prime}\right) \in \mathcal{R}_{(S, \leq)}(A)$ and $\left(p_{A}^{\prime}, p_{A}\right) \in \mathcal{R}_{(S, \leq)}(A)$ implies $p_{A}=p_{A}^{\prime}$ in $\pi_{S}(A)$;

(4) if $\left(p_{A}, p_{A}^{\prime}\right) \in \mathcal{R}_{(S, \leq)}(A)$ and $\varphi \in \operatorname{Mor}_{\mathcal{C}}(B, A)$ then $\left(p_{A} \circ \varphi, p_{A}^{\prime} \circ \varphi\right) \in$ $\mathcal{R}_{(S, \leq)}(B)$.

The functor acts on morphisms by $\mathcal{R}_{(S, \leq)}(\varphi: B \rightarrow A): \mathcal{R}_{(S, \leq)}(A) \rightarrow \mathcal{R}_{(S, \leq)}(B)$ mapping $\left(p_{A}, p_{A}^{\prime}\right) \mapsto\left(p_{A} \circ \varphi, p_{A}^{\prime} \circ \varphi\right)$.

3.2. Sublevel sieve. In this general setting, instead of the sublevel sets and sublevel objects we considered in the previous sections, we construct sublevels as subfunctors of the functor of points.

3.2.1. Lemma. (i) Let $\mathcal{C}$ be a category with terminal object $\star$. Let $(S, \leq)$ be a poset functor on an object $S \in \operatorname{Obj}(\mathcal{C})$ as in Definition 3.1.1.

Starting with a family $f: X \rightarrow S$ in $\mathcal{C}$ and a choice of $s \in \operatorname{Mor}_{\mathcal{C}}(\star, S)$, consider the assignment

$$
X_{f, \leq, s}(A)=\left\{\alpha \in \pi_{X}(A)=\operatorname{Mor}_{\mathcal{C}}(A, X): f \circ \alpha \leq s \circ t_{A}\right\}
$$

where $t_{A}: A \rightarrow \star$ is the unique morphism to the terminal object in $\mathcal{C}$ and $f \circ \alpha \leq$ $s \circ t_{A}$ means that $\left(f \circ \alpha, s \circ t_{A}\right) \in \mathcal{R}_{(S, \leq)}(A) \subset \pi_{S}(A) \times \pi_{S}(A)$.

Given a morphism $\varphi: B \rightarrow A$ in $\operatorname{Mor}_{\mathcal{C}}(B, A)$ assign to it the map

$$
X_{f, \leq, s}(\varphi: B \rightarrow A): X_{f, \leq, s}(A) \rightarrow X_{f, \leq, s}(B), \quad \alpha \mapsto \alpha \circ \varphi
$$

This assignment determines a contravariant functor $X_{f, \leq, s}: \mathcal{C} \rightarrow$ Sets, the "sublevel functor" of $f: X \rightarrow S$. 
(ii) For all $s \in \operatorname{Mor}_{\mathcal{C}}(\star, S)$ the sublevel functor $X_{f, \leq, s}$ is a subfunctor of the functor of points $\pi_{X}$. Moreover, for $s \leq s^{\prime}$ in $\operatorname{Mor}_{\mathcal{C}}(\star, S)$, the sublevel functor $X_{f, \leq, s}$ is a subfunctor of $X_{f, \leq, s^{\prime}}$.

Proof. (i) By Definition 3.1.1, the subsets $\mathcal{R}_{(S, \leq)}(A) \subset \pi_{S}(A) \times \pi_{S}(A)$ have the following property: if $\left(f \circ \alpha, s \circ t_{A}\right) \in \mathcal{R}_{(S,<)}(A)$ then for any $\varphi: B \rightarrow A$ in $\operatorname{Mor}_{\mathcal{C}}(B, A)$ the element $\left(f \circ \alpha \circ \varphi, s \circ t_{A} \circ \varphi\right) \in \mathcal{R}_{(S, \leq)}(B)$, where $t_{A} \circ \varphi=t_{B}$ is the unique morphism $t_{B}: B \rightarrow \star$ to the terminal object. Thus, the assignment above is well defined and determines a contravariant functor.

(ii) For all $A \in \operatorname{Obj}(\mathcal{C})$ by construction we have $X_{f, \leq, s}(A) \subseteq \pi_{X}(A)$. Moreover, for a morphism $\varphi: B \rightarrow A$ the image $X_{f,<, s}(\varphi)$ is the restriction of $\pi_{X}(\varphi)$ (precomposition with $\varphi$ ) to $X_{f, \leq, s}(B)$. Hence $X_{f, \leq, s}$ is a subfunctor of the functor $\pi_{X}$. Similarly, if $s \leq s^{\prime}$, that is $\left(s, s^{\prime}\right) \in \mathcal{R}_{(S, \leq)}(\star)$, the condition $f \circ \alpha \leq s \circ t_{A}$ implies that $f \circ \alpha \leq s^{\prime} \circ t_{A}$ hence $X_{f, \leq, s}(A) \subseteq X_{f, \leq, s^{\prime}}(A)$ and $X_{f, \leq, s}(\varphi)$ is the restriction of $X_{f, \leq, s^{\prime}}(\varphi)$ hence $X_{f, \leq, s}$ is a subfunctor of $X_{f, \leq, s^{\prime}}$. This completes the proof.

An assignment of a subfunctor of the functor of points $\pi_{X}$ is a sieve on $X$. Thus, we equivalently refer to $X_{f, \leq, s}$ as the sublevel sieve of $X$.

3.2.2. Definition. If the subfunctor $X_{f, \leq, s}$ of the functor of points $\pi_{X}$ is representable, the object $X_{s} \in \operatorname{Obj}(\mathcal{C})$ with $X_{f, \leq, s}(A)=\operatorname{Mor}_{\mathcal{C}}\left(A, X_{s}\right)$ is the " $s$ sublevel object" of $X$.

Cases where the sublevel functor $X_{f, \leq, s}$ is representable include geometric cases where it is a closed subfunctor.

More precisely, if the sublevel functor $X_{f, \leq, s}$ is representable by an object $X_{s} \in$ $\operatorname{Obj}(\mathcal{C})$, then for any $s, s^{\prime} \in \operatorname{Mor}_{\mathcal{C}}(\star, S)$ with $s \leq s^{\prime}$ there is a monomorphism $j_{s, s^{\prime}}: X_{s} \hookrightarrow X_{s^{\prime}}$, since the inclusions $X_{f, \leq, s}(A) \subseteq X_{f, \leq, s^{\prime}}(A)$ are monomorphisms of sets $j_{s, s^{\prime}}: \pi_{X_{s}} \rightarrow \pi_{X_{s^{\prime}}}$ which induce corresponding morphisms $X_{s} \rightarrow X_{s^{\prime}}$ in $\mathcal{C}$ with the property that for all $u, v \in \pi_{X_{s}}$ if $j_{s, s^{\prime}} \circ u=j_{s, s^{\prime}} \circ v$ then $u=v$, hence $j_{s, s^{\prime}}$ is a monomorphism in $\mathcal{C}$. In the case of a representable sublevel functor we can define persistent functors in the following way.

3.2.3. Definition. Let $\mathcal{C}$ be a category as above with terminal object $\star$ and $(S, \leq)$ be a poset functor on an object $S \in \operatorname{Obj}(\mathcal{C})$. Let $\mathcal{H}: \mathcal{C} \rightarrow \mathcal{A}$ be a covariant functor to an abelian category. For $f: X \rightarrow S$, and $s \in \operatorname{Mor}_{\mathcal{C}}(\star, S)$ let $X_{f, \leq, s}$ be the sublevel functor. If $X_{f, \leq, s}$ is representable by $X_{s} \in \operatorname{Obj}(\mathcal{C})$, then the persistent functor $P \mathcal{H}$ is given by

$$
P \mathcal{H}_{\left(s, s^{\prime}\right)}(X)=\text { Range }\left(\mathcal{H}\left(j_{s, s^{\prime}}\right): \mathcal{H}\left(X_{s}\right) \rightarrow \mathcal{H}\left(X_{s^{\prime}}\right)\right) \quad \text { for } s \leq s^{\prime}
$$


Return to the category $\tilde{\mathcal{C}}_{S}$ of families in $\mathcal{C}$. For $(S, \leq)$ as above and $s \in$ $\operatorname{Mor}_{\mathcal{C}}(\star, S)$, and for an abelian category $\mathcal{A}$, we define $\mathcal{A}^{(S, \leq)}$ as the category of covariant functors $F: \mathcal{R}_{(S, \leq)}(\star) \rightarrow \mathcal{A}$ and natural transformations of such functors. We can then interpret, for fixed $s^{\prime} \in \operatorname{Mor}_{\mathcal{C}}(\star, S)$ the persistent functor $P \mathcal{H}$ as a functor $P \mathcal{H}_{s^{\prime}}: \tilde{\mathcal{C}} \rightarrow \mathcal{A}^{(S, \leq)}$ with

$$
P \mathcal{H}_{s^{\prime}}(X, f)=s \mapsto P \mathcal{H}_{\left(s, s^{\prime}\right)}(X)
$$

for $s \leq s^{\prime}$ and zero otherwise. For a morphism $\varphi:(X, f) \rightarrow\left(X^{\prime}, f^{\prime}\right)$ we define $P_{\mathcal{H}^{\prime}}\left(\varphi:(X, f) \rightarrow\left(X^{\prime}, f^{\prime}\right)\right)$ the map induced on Range $\left(\mathcal{H}\left(j_{s, s^{\prime}}\right): \mathcal{H}\left(X_{s}\right) \rightarrow\right.$ $\left.\mathcal{H}\left(X_{s^{\prime}}\right)\right)$ by $\mathcal{H}(\varphi): \mathcal{H}\left(X_{s}\right) \rightarrow \mathcal{H}\left(X_{s}^{\prime}\right)$

\section{NORI DIAGRAMS AND TANNAKIAN FORMALISM}

4.1. Setup. Start with a diagram $D$ and an $R$-linear abelian category $\mathcal{A}$, where $R$ is a commutative ring. Consider a representation $T: D \rightarrow \mathcal{A}^{(S, \leq)}$, where $(S, \leq)$ is a thin category, and $\mathcal{A}^{(S, \leq)}=\mathcal{F}((S, \leq), \mathcal{A})$ is the category of covariant functors from a thin category $(S, \leq)$ to $\mathcal{A}$.

The representation $T$ assigns to each vertex $v \in V(D)$ a functor $T(v):(S, \leq) \rightarrow$ $\mathcal{A}$ and to each edge $e \in E(D)$ a natural transformation $T(e): T(s(e)) \rightarrow T(t(e))$ between the functors associated to the source $s(e)$ and target $t(e)$ vertices of $e$.

For a vertex $v \in V(D)$, denote by $\mathcal{N}_{v}$ be the set of natural self-transformations $\alpha_{v}: T(v) \rightarrow T(v)$ of the functors $T(v):(S, \leq) \rightarrow \mathcal{A}$. Moreover, put

$$
\mathcal{N}(T)=\left\{\left(\alpha_{v}\right)_{v \in V(D)}: \alpha_{v} \in \mathcal{N}_{v} \text { with } \alpha_{t(e)} \circ T(e)=T(e) \circ \alpha_{s(e)}, \forall e \in E(D)\right\}
$$

Generally, given an abelian category $\mathcal{B}$ and a set $\mathcal{S}$ of objects in $\mathcal{B}$. As in $[\mathrm{HuM}-$ S17], denote by $\langle\mathcal{S}\rangle$ the smallest full abelian subcategory of $\mathcal{B}$ that contains $\mathcal{S}$ and such that the inclusion functor is exact. It is generated by the objects in $\mathcal{S}$ and is closed under taking direct sums, direct summands, kernels and cokernels.

Notice that here we do not assume that we start with a representation of the diagram $D$ in a category of $R$-modules for some ring $R$. Hence we do not have

an obvious choice of a faithful exact functor from $\mathcal{N}(T)$-Mod to $\mathcal{A}^{(S, \leq)}$ playing the role of the forgetful functor to $R$-Mod in the setting of [HuM-S17]. However, we can still construct an abelian category $\mathcal{C}\left(D, T, \mathcal{A}^{(S, \leq)}\right)$ associated to the data 
$\left(D, T, \mathcal{A}^{(S, \leq)}\right)$ with the property that the representation $T: D \rightarrow \mathcal{A}^{(S, \leq)}$ factors through $\mathcal{C}\left(D, T, \mathcal{A}^{(S, \leq)}\right)$.

4.1.1. Lemma. Let $T: D \rightarrow \mathcal{A}^{(S, \leq)}$ be a diagram representation as above. Consider the abelian subcategory $\langle T(D)\rangle$ of $\mathcal{A}^{(S, \leq)}$. There is an inclusion functor $\langle T(D)\rangle \hookrightarrow \mathcal{N}(T)$-Mod. Let $\mathcal{C}\left(D, T, \mathcal{A}^{(S, \leq)}\right)$ denote the subcategory of $\mathcal{N}(T)$-Mod obtained in this way. If the inclusion functor $\langle T(D)\rangle \hookrightarrow \mathcal{N}(T)$-Mod is exact, this is an abelian subcategory. Moreover, there is a representation $\tilde{T}: D \rightarrow \mathcal{C}\left(D, T, \mathcal{A}^{(S, \leq)}\right)$ such that $T$ factors as $T=F \circ \tilde{T}$ with a faithful exact functor $F: \mathcal{C}\left(D, T, \mathcal{A}^{(S, \leq)}\right) \rightarrow$ $\mathcal{A}^{(S, \leq)}$.

Proof. Since $\mathcal{A}$ is an $R$-linear abelian category, the category $\mathcal{A}^{(S, \leq)}$ is also an $R$-linear abelian category (Proposition 44 of [Mu06]), and the sets $\mathcal{N}_{v}$ and $\mathcal{N}(T)$ are rings with respect to the composition operation. We proceed as in Proposition 7.3.24 of [HuM-S17], assuming for simplicity that $D$ is a finite diagram.

Consider the object $X=\oplus_{v} T(v)$. Then $\langle T(D)\rangle=\langle X\rangle$. Let $\mathcal{N}_{X}$ be the set of natural self-transformations of the functor $X:(S, \leq) \rightarrow \mathcal{A}$. Among the transformations in $\mathcal{N}_{X}$ we can identify the elements of $\mathcal{N}(T)$ as those $\alpha \in \mathcal{N}_{X}$ that commute with the projections $p_{v}: X \rightarrow T(v)$ and with the transformations $T(e)$. Thus, we can view $\langle T(D)\rangle$ as a subcategory of $\mathcal{N}(T)$-Mod. If the inclusion functor $\langle T(D)\rangle \hookrightarrow \mathcal{N}(T)$-Mod is an exact functor then $\langle T(D)\rangle$ gives an abelian subcategory of $\mathcal{N}(T)$-Mod, which we denote by $\mathcal{C}\left(D, T, \mathcal{A}^{(S, \leq)}\right)$. There is a representation $\tilde{T}: D \rightarrow \mathcal{C}\left(D, T, \mathcal{A}^{(S, \leq)}\right)$ given by the assignment $v \mapsto T(v), e \mapsto T(e)$ of the representation $T: D \rightarrow \mathcal{A}^{(S, \leq)}$, seen as objects of $\langle T(D)\rangle$. By construction, this representation satisfies $T=F \circ \tilde{T}$, where $F: \mathcal{C}\left(D, T, \mathcal{A}^{(S, \leq)}\right) \rightarrow\langle T(D)\rangle \hookrightarrow \mathcal{A}^{(S, \leq)}$ is the forgetful functor that forgets the $\mathcal{N}(T)$-module structure.

This completes the proof.

4.1.2. Remark. If the abelian category $\mathcal{A}$ has a tensor structure and $\mathcal{A}^{(S, \leq)}$ is endowed with the pointwise tensor structure, and we assume that the diagram $D$ is a graded diagram with a commutative product with unit, we can consider representations $T: D \rightarrow \mathcal{A}^{(S, \leq)}$ that are unital graded multiplicative representations so that $\mathcal{C}\left(D, T, \mathcal{A}^{(S, \leq)}\right)$ also has a natural tensor structure such that $F: \mathcal{C}\left(D, T, \mathcal{A}^{(S, \leq)}\right) \rightarrow \mathcal{A}^{(S, \leq)}$ becomes a tensor functor. (The argument for the original setting of diagram representations to $R$-Mod is given in Proposition 8.1.5(1) of [HuM-S17].) We can then consider faithful exact tensor functors from $\mathcal{C}\left(D, T, \mathcal{A}^{(S, \leq)}\right)$ to categories $\mathcal{B}^{(S, \leq)}$, where $\mathcal{B}$ is an $R$-linear abelian tensor category and $\mathcal{B}^{(S, \leq)}$ is endowed with the pointwise tensor structure. In particu- 
lar, one can study tensor functors $\mathcal{C}\left(D, T, \mathcal{A}^{(S, \leq)}\right) \rightarrow \operatorname{Vec}^{(S, \leq)}$, as generalizations of persistent homology.

\section{PERSISTENCE OF NORI MOTIVES}

We return here to the algebraic geometric environment of Nori motives, in its updated form of Arapura's category of Nori Motivic Sheaves, [Ar13]. We enrich it with a Persistence structure. We write here the Nori motives covariantly (homologically) as in [Ar13], rather than contravariantly (cohomologically) as in the initial Grothendieck's project and in [HuM-S17].

Let $S$ be a connected variety over $\mathbf{K}$. The Nori Diagram $D_{m s}(S)$ for Motivic Sheaves over a base $S$ has the following structure (]Ar13]):

- One vertex in $V\left(D_{m s}(S)\right)$ is a quadruple $(f: X \rightarrow S, Y, k, w)$, where $X$ is a K-variety with a morphism $f: X \rightarrow S ; j: Y \hookrightarrow X$ is a closed embedding (endowed with the restriction $\left.\left.f\right|_{Y}: Y \rightarrow S\right), k \in \mathbf{Z}_{+}$is a non-negative integer. and $w \in \mathbf{Z}$ is an integer.

- Edges in $E\left(D_{m s}(S)\right)$ are of the following types:

(1) Geometric morphisms: each morphism of varieties $\varphi: X \rightarrow X^{\prime}$ compatible with the maps to the base $S$ and the inclusions via commutative diagrams
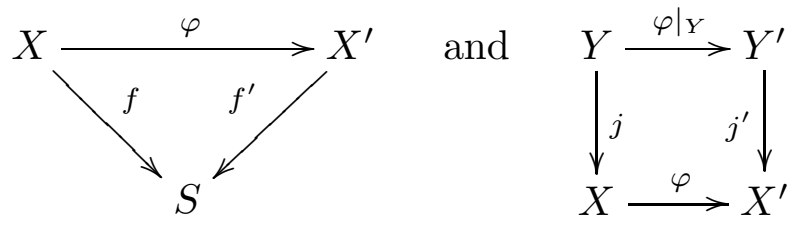

produces an edge

$$
(f: X \rightarrow S, Y, k, w) \rightarrow\left(f^{\prime}: X^{\prime} \rightarrow S, Y^{\prime}, k, w\right) .
$$

(2) Connecting morphisms: every chain of closed embeddings $Z \subset Y \subset X$ determines an edge

$$
(f: X \rightarrow S, Y, k+1, w) \rightarrow\left(\left.f\right|_{Y}: Y \rightarrow S, Z, k, w\right)
$$

(3) Twist morphisms: for every vertex $(f: X \rightarrow S, Y, k, w)$ there is an edge

$$
\left(f \circ p_{1}: X \times \mathbf{P}^{1} \rightarrow S, Y \times \mathbf{P}^{1} \cup X \times\{0\}, k+2, w+1\right) \rightarrow(f: X \rightarrow S, Y, k, w)
$$


A representation of the diagram $D_{m s}(S)$ is given by the constructible sheaves

$$
T_{m s}:(f: X \rightarrow S, Y, k, w) \mapsto H_{S}^{k}(X, Y, \mathbf{F})
$$

The category of Nori Motivic Sheaves is defined as the Nori Diagram Category $\mathcal{C}\left(D_{m s}(S), T_{m s}\right)$ of this representation.

5.1. Posets and semigroups. In order to enrich this construction with a persistent version of the constructible sheaves $H_{S}^{k}(X, Y, \mathbf{F})$, we follow the general formalism described in the previous sections. The first step is to consider a base $S$ that is endowed with a poset structure that will provide the indexing of the persistence modules.

A source of poset structures on geometric spaces such as $\mathbf{K}$-varieties is the presence of a semigroup structure. Indeed there are natural poset structures associated to semigroups, which we review briefly, see [Mi86].

Given a semigroup $S$, the set $E_{S}$ of idempotents $e \in S, e^{2}=e$, is partially ordered by the relation $e \leq e^{\prime}$ if $e=e e^{\prime}=e^{\prime} e$. A natural partial order structure on a semigroup is one that restricts to this relation on the set of idempotents. Recall that a semigroup $S$ is regular if every element $s \in S$ has a pseudoinverse $x$ such tat $s x s=s$.

The Nambooripad poset structure on a regular semigroup ([Na80]) is defined by

$$
s \leq s^{\prime} \quad \text { iff } s=e s^{\prime}=s^{\prime} e^{\prime}, \text { for some } e, e^{\prime} \in E_{S} .
$$

Several equivalent definitions are discussed in [Mi86]. This is further extended to more general semigroups as shown in [Mit86] as follows. Let $\hat{S}$ be obtained by adjoining a unit to the semigroup $S$ (or $S$ itself if it already has a unit). If $E_{S}$ is a subsemigroup of $S$ then the relation $s \leq s^{\prime}$ iff $s=e s^{\prime}=s^{\prime} e^{\prime}$ for some $e, e^{\prime} \in E_{\hat{S}}$ is a partial order compatible with multiplication. On an arbitrary semigroup the relation

$$
s \leq s^{\prime} \text { iff } s=x s^{\prime}=s^{\prime} y \text { and } x s=s \text { for some } x, y \in \hat{S}
$$

is a partial order, which we call as the natural partial order (see Proposition 2 and Theorem 3 of [Mi86]).

5.2. Varieties with semigroup structures. In order to obtain a base $S$ with a poset structure $(S, \leq)$ it is then sufficient to consider the case where $S$ 
has a semigroup structure. Semigroup structures on algebraic varieties have been investigated in [Br14a]. We review here some general facts from [Br14a] and [Br14b] and some examples from [Br14a].

We consider varieties over an algebraically closed field $\mathbf{K}$. Let $S$ be an algebraic semigroup, that is, an algebraic variety over $\mathbf{K}$ endowed with semigroup operation. Let $E_{S}$ be the subscheme of idempotents of $S$. The partial order structure on idempotents in $E_{S}$ is given by $e_{0} \leq e_{1}$ iff $e_{0}=e_{0} e_{1}=e_{1} e_{0}$. Given two idempotents $e_{0}, e_{1}$ with $e_{0} \leq e_{1}$, the "interval" $\left[e_{0}, e_{1}\right]$ is given by

$$
\left[e_{0}, e_{1}\right]:=\left\{e \in E_{S}: e_{0} \leq e \leq e_{1}\right\}
$$

and is a closed subscheme of $S$ ([Br14b], Corollary 2.17). If $S$ is a smooth algebraic semigroup, then the scheme $E_{S}$ of idempotents is also smooth ([Br14b], Remark 2.15). If $S$ is a commutative algebraic semigroup, then the scheme $E_{S}$ of idempotents is finite and reduced ([Br14b], Theorem 1.2). If $S$ is irreducible, then there is a smallest closed and irreducible subsemigroup of $S$ that contains $E_{S}$, which is given by a toric monoid ([Br14b], Theorem 1.2).

Classes of examples of algebraic varieties with semigroup structures include:

- linear algebraic semigroups: subsemigroups of $\operatorname{End}(V)$ with $V$ some finite dimensional vector space;

- an arbitrary variety $S$ with the left/right projection semigroup laws $\mu_{L}(x, y)=$ $x$ and $\mu_{R}(x, y)=y$ for all $x, y \in X$;

- algebraic groups;

- algebraic semigroup laws on Abelian varieties classified in Section 4 of [Br14a].

- algebraic semigroup laws on affine monomial curves (Theorem 5 of [Br14a]).

5.3. Sublevel subschemes over semigroups. We consider the cases where $E_{S}$ is a subsemigroup of $S$ so that $S$ has a Nambooripad poset structure.

5.3.1. Lemma. Let $S$ be an algebraic semigroup, such that the idempotent subscheme $E_{S}$ is a subsemigroup of $S$. Let $(S, \leq)$ be the Nambooripad poset structure. For a morphism $f: X \rightarrow S$, the sublevel sets $X_{s}=\{x \in X: f(x) \leq s\}$ are closed subschemes of $X$.

Proof. The subset $S_{s}=\{a \in S: a \leq s\}$ is given by all the elements $a \in S$ that are of the form $a=e s=s e^{\prime}$ for some pair $\left(e, e^{\prime}\right) \in E_{S}^{2}$. Thus, we have $S_{s}=E_{S} \cdot s \cap s \cdot E_{S} \subset S$. For an algebraic semigroup, the subscheme $E_{S}$ of 
idempotents is closed, hence for a fixed $s \in S$ we obtain $S_{a}$ as an intersection of two closed subschemes. The sublevel sets $X_{s}=f^{-1}\left(S_{s}\right)$ are then preimages in $X$ of closed subschemes of $S$. This completes the proof.

The sublevel subvarieties $X_{s}$ have embeddings $j_{s, s^{\prime}}: X_{s} \hookrightarrow X_{s^{\prime}}$ for $s \leq s^{\prime}$ due to the transitive property of the partial order relation on $S$.

5.3.2. Corollary. If $S$ is a commutative algebraic semigroup, which is smooth as an algebraic variety, and $(S, \leq)$ is the Nambooripad poset structure, then the sublevels $X_{s}$ of a morphism $f: X \rightarrow S$ are finite unions of fibers.

Proof. For a commutative smooth algebraic semigroup $S$, the idempotent subscheme $E_{S}$ is a subvariety of $S$ consisting of a finite set of points. Then the $S_{s}$ are also finite and identified with $\left\{e s e \in E_{S}\right\}$, and the preimages $X_{s}=f^{-1}\left(S_{s}\right)=$ $\cup_{e \in E_{S}} f^{-1}(e s)$ are finite unions of fibers of the morphism $f: X \rightarrow S$.

5.4. Persistent Nori Motivic Sheaves. Let $S$ be a $\mathbf{K}$-variety with a semigroup structure and an associated partial order $(S, \leq)$ as discussed above. As in Arapura's setting of Nori Motivic Sheaves in [Ar13] we consider the Nori Diagram with vertices $(f: X \rightarrow S, Y, k, w)$ and with the three classes of edges described above. To this diagram $D_{m s}(S, \leq)$ we associate a representation in the abelian category $\operatorname{Vec}^{(S, \leq)}$ in the following way.

We consider a Persistence Diagram $D_{m s}^{P}(S, \leq)$ of Motivic Sheaves over a base algebraic semigroup $S$ with an associated partial order $\leq$ determined by the semigroup structure with the following vertices and edges:

- Vertices in $V\left(D_{m s}^{P}(S, \leq)\right)$ are given by elements of the form $(f: X \rightarrow S, Y, k, w, s)$, where $X$ is a $\mathbf{K}$-variety with a morphism $f: X \rightarrow S, j: Y \hookrightarrow X$ is a closed embedding (endowed with the restriction $\left.f\right|_{Y}: Y \rightarrow S$ ), $k \in \mathbf{Z}_{+}$is a non-negative integer, $w \in \mathbf{Z}$ is an integer, and $s \in S$.

- Edges in $E\left(D_{m s}^{P}(S, \leq)\right)$ are of the following types:

(1) Geometric morphisms: for a morphism of varieties $\varphi: X \rightarrow X^{\prime}$ compatible with the maps to the base $S$ and the inclusions via commutative diagrams
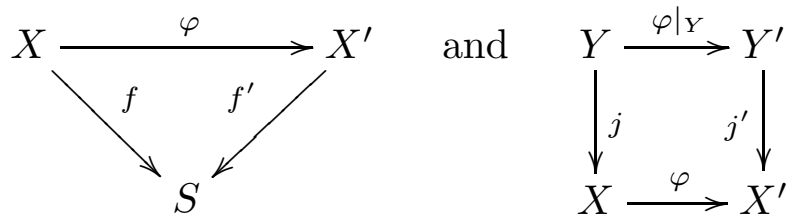
there corresponds an edge

$$
(f: X \rightarrow S, Y, k, w, s) \rightarrow\left(f^{\prime}: X^{\prime} \rightarrow S, Y^{\prime}, k, w, s\right)
$$

(2) Connecting morphisms: every chain of closed embeddings $Z \subset Y \subset X$ determines an edge

$$
(f: X \rightarrow S, Y, k+1, w, s) \rightarrow\left(\left.f\right|_{Y}: Y \rightarrow S, Z, k, w, s\right)
$$

(3) Twist morphisms: for every vertex $(f: X \rightarrow S, Y, k, w)$ there is an edge

$$
\left(f \circ p_{1}: X \times \mathbf{P}^{1} \rightarrow S, Y \times \mathbf{P}^{1} \cup X \times\{0\}, k+2, w+1, s\right) \rightarrow(f: X \rightarrow S, Y, k, w, s)
$$

(4) Persistence morphisms: for all $s^{\prime} \in S$ with $s \leq s^{\prime}$ an edge

$$
(f: X \rightarrow S, Y, k, w, s) \rightarrow\left(f: X \rightarrow S, Y, k, w, s^{\prime}\right)
$$

A representation of the diagram $D_{m s}^{P}(S, \leq)$ in $\operatorname{Vec}^{(S, \leq)}$ is then obtained as follows. Consider the map that assigns to each vertex $\left(f: X \rightarrow S, Y, k, w,\left(s, s^{\prime}\right)\right)$ the functor

$$
F:(S, \leq) \rightarrow \text { Vec }
$$

where we view $(S, \leq)$ as a thin category, given by

$$
F(t)=\operatorname{Range}\left(H_{*}\left(j_{t, s}\right): H_{*}\left(X_{t}, Y_{t} ; \mathbf{Q}\right) \rightarrow H_{*}\left(X_{s}, Y_{s} ; \mathbf{Q}\right)\right),
$$

for all $t \leq s$ in $S$ and zero otherwise. The edges listed above are correspondingly mapped to homomorphisms of the homology groups $H_{*}\left(X_{t}, Y_{t} ; \mathbf{Q}\right)$ and $H_{*}\left(X_{s}, Y_{s} ; \mathbf{Q}\right)$ with induced morphisms on Range $\left(H_{*}\left(j_{t, s}\right)\right)$.

\section{MODEL CATEGORIES AND PERSISTENT TOPOLOGY}

In this section we address a question that was posed to us by Jack Morava, about developing a suitable model structure for persistent topology.

6.1. Model categories. We review quickly some basic definitions regarding model structures and categories that we will be using in the following. 
Recall that a morphism $f$ in a category is called a retract of another morphism $g$ iff there is a commutative diagram

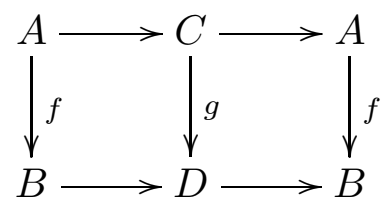

where the horizontal compositions are the identities.

A model category $\mathcal{M}$ is a category together with three classes of morphisms: weak equivalences, fibrations and cofibrations. These data must satisfy the following axioms:

(1) $\mathcal{M}$ has all small limits and colimits;

(2) if in a composition $g \circ f$ of morphisms two among the three maps $f, g, g \circ f$ are weak equivalences, then the third also is;

(3) if a map $f$ is a retract of $g$, and if $g$ is a weak equivalence, fibration, or cofibration, then $f$ also is;

(4) given a commutative diagram

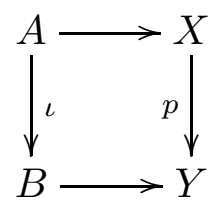

a lift $B \rightarrow X$ exists if either $\iota$ is a cofibration and $p$ is an acyclic fibration (i.e. both a fibration and a weak equivalence) or if $\iota$ is an acyclic cofibration (i.e. both a cofibration and a weak equivalence) and $p$ is a fibration;

(5) morphisms $g$ in $\mathcal{M}$ can be factored as $g=q i$ with $q$ an acyclic fibration and $i$ a cofibration or as $g=p j$ with $p$ a fibration and $j$ an acyclic cofibration.

Let $\mathcal{M}, \mathcal{N}$ be model categories. A Quillen pair $L: \mathcal{M} \leftrightarrow \mathcal{N}: R$ is an adjoint pair of functors $(L, R)$ where $L$ preserves cofibrations and $R$ preserves fibrations, see Section 1.6 of [Be18].

A model category $\mathcal{M}$ is cofibrantly generated iff: 
(1) there is a set $I$ of cofibrations of $\mathcal{M}$ such that the domains of the elements of $\mathcal{I}$ are small with respect to $\mathcal{I}$ and such that a map is an acyclic fibration iff it has the right lifting property with respect to $\mathcal{I}$;

(2) there is a set $\mathcal{J}$ of acyclic cofibrations of $\mathcal{M}$ (also with the property that the domains are small with respect to $\mathcal{J}$ ) such that a map is a fibration iff it has the right lifting property with respect to $\mathcal{J}$.

The set $\mathcal{I}$ is the set of generating cofibrations and the set $\mathcal{J}$ is the set of generating acyclic cofibrations (see Section 1.7 of [Be18] for the terminology and for more details).

A model category $\mathcal{M}$ is called combinatorial if it is cofibrantly generated and as a category it is locally presentable, that is, it admits all small colimits and a set of small objects such that any object can be obtained as colimit of a small diagram with objects in this set, see Sec. 2.7 of [Be18] for a more detailed exposition.

6.2. Model structures on categories of functors. Given a small category $\mathcal{C}$ and a category $\mathcal{D}$, we denote by $\mathcal{F}(\mathcal{C}, \mathcal{D})$ the category of functors, whose objects are the covariant functors $F: \mathcal{C} \rightarrow \mathcal{D}$ and morphisms the natural transformations of these functors.

For example, the categories we considered in the previous sections of the form $\operatorname{Vec}^{(S, \leq)}$ with $(S, \leq)$ a thin category are categories of functors, and so are the $\operatorname{Top}^{(S, \leq)}$ considered in [BuSc14], [BuSiSc15] as a setting for persistent topology.

It is known that if the category $\mathcal{D}$ has a model structure that is cofibrantly generated, then for any small category $\mathcal{C}$ the category of functors $\mathcal{F}(\mathcal{C}, \mathcal{D})$ also has a model structure, called the projective model structure, which is also cofibrantly generated, see Sec. 11.6 of [HI03]. If the category $\mathcal{D}$ has a combinatorial model structure, then the category of functors $\mathcal{F}(\mathcal{C}, \mathcal{D})$ has a model structure, called the injective model structure.

In the projective model structure on $\mathcal{F}(\mathcal{C}, \mathcal{D})$ weak equivalences and projective fibrations are those natural transformations $\eta: F \rightarrow F^{\prime}$ of functors $F, F^{\prime}: \mathcal{C} \rightarrow \mathcal{D}$ such that for all objects $X \in \operatorname{Obj}(\mathcal{C})$, the morphisms $\eta_{X}: F(X) \rightarrow F^{\prime}(X)$ in $\mathcal{D}$ are, respectively, weak equivalences and fibrations. Similarly, in the injective model structure injective weak equivalences and injective cofibrations are natural transformations that are, object-wise in $\mathcal{C}$, weak equivalences and cofibrations in $\mathcal{D}$.

6.3. Model structure for datasets and Vietoris-Rips complexes. We consider here the main example of persistent topology, which accounts for its use 
in topological data analysis, namely datasets with their associated Vietoris-Rips complexes, and their persistent homology barcode diagrams.

We construct a model category for this setting in several steps:

(1) We start by considering the model structure on the categories of simplicial sets $\Delta \mathcal{S}$ and of chain complexes $\mathrm{Ch}_{R}$ over a commutative ring $R$. We denote by $\mathcal{M}$ either of these model categories.

(2) We induce a projective model structure on the category of functors

$$
\mathcal{M}^{(S, \leq)}=\mathcal{F}((S, \leq), \mathcal{M})
$$

where $(S, \leq)$ is a suitable poset, viewed as a thin category.

(3) We construct a category $\mathcal{P}_{\mathbf{E}}$ of finitely supported probability distributions in a fixed ambient metric space $\mathbf{E}$ (e. g. an Euclidean space of sufficiently large dimension).

(4) We describe the assignment of Vietoris-Rips complexes to datasets as a functor $V R: \mathcal{P}_{\mathbf{E}} \rightarrow \mathcal{M}^{(S, \leq)}$.

(5) We use Dugger's construction [Du01] of a universal model category associated to a small category to construct a model category $U\left(\mathcal{P}_{\mathbf{E}}\right)$ for finitely supported probability distributions with a Vietoris-Rips functor $V R: U\left(\mathcal{P}_{\mathbf{E}}\right) \rightarrow \mathcal{M}^{(S, \leq)}$.

6.3.1. Model structure on chain complexes. Let $\mathrm{Ch}_{R}$ be the category of (unbounded) chain complexes over a commutative ring $R$ with chain maps as morphisms. The weak equivalences are the quasi-isomorphisms of chain complexes. The fibrations are the chain maps $\varphi_{\bullet}: C_{\bullet} \rightarrow C_{\bullet}^{\prime}$ such that for each level $n$ the map $\varphi_{n}: C_{n} \rightarrow C_{n}^{\prime}$ is an epimorphism of $R$-modules. The cofibrations are chain maps that are level-wise monomorphisms of $R$-modules with projective cokernel.

This is a projective model structure on $\mathrm{Ch}_{R}$. One can similarly consider the injective model structure with the same weak equivalences, but with cofibrations given by chain maps that are level-wise injective morphisms of $R$-modules, while fibrations are level-wise epimorphisms with injective kernel.

These two model structures are Quillen-equivalent (Section 1.7 of [Be18]). The projective model structure on $\mathrm{Ch}_{R}$ is cofibrantly generated (see Sec. 2.3.11 of [Hov98] and Sec. 5 of [SchSh00]).

6.3.2. Model structure on simplicial sets. The category of simplicial sets $\Delta \mathcal{S}$ has a model structure (the Kan-Quillen model structure) where the weak equivalences are morphisms that induce a weak homotopy equivalence of topological 
spaces at the level of geometric realizations, the fibrations are Kan fibrations, and the cofibrations are monomorphisms of simplicial sets. The Kan-Quillen model structure is cofibrantly generated, with generating cofibrations the boundary inclusions and generating acyclic cofibrations the horn inclusions, see [Be18], [GeMa03], [GoJa99], [Hi03].

6.3.3. Model structure on indexed diagrams. Let $\mathcal{M}$ denote either the category of chain complexes $\mathrm{Ch}_{R}$ with the projective model structure, or the category of simplicial sets $\Delta \mathcal{S}$ with the Kan-Quillen model structure. We consider now the category $\mathcal{M}^{(S, \leq)}$ of $(S, \leq)$-indexed diagrams in $\mathcal{M}$, for a poset $(S, \leq)$. This is the same as the category

$$
\mathcal{M}=\mathcal{F}((S, \leq), \mathcal{M})
$$

of covariant functors from the thin category $(S, \leq)$ to $\mathcal{M}$ with morphisms given by natural transformations. This will include the case of $\mathcal{M}^{(\mathbf{R}, \leq)}$ that will correspond to the usual Vietoris-Rips complexes of data sets, but we will work with a more general $(S, \leq)$ that also incorporates in the Vietoris-Rips complex a cutoff according to a probability (see Part IV of [BoChYv18]).

Since the model category $\mathcal{M}$ is cofibrantly generated, the category of functors $\mathcal{M}^{(S, \leq)}$ admits a projective model structure that is also cofibrantly generated.

6.3.4. A category of data sets. Consider the following small category $\mathcal{P}_{\mathbf{E}}$. Its objects are triples $(A, f, P)$ of a finite set $A$, an embedding $f: A \hookrightarrow \mathbf{E}$ in a fixed ambient metric space $\mathbf{E}$, which we can assume to be an Euclidean space of some fixed sufficiently large dimension, and a probability distribution $P$ on $A$, which we can view as a probability on $\mathbf{E}$ supported on the finite set $f(A)$ through pushforward along the map $f$.

A morphism in $\operatorname{Mor}_{\mathcal{P}_{\mathbf{E}}}\left((A, f, P),\left(A^{\prime}, f^{\prime}, P^{\prime}\right)\right)$ is a pair $(\varphi, \tilde{\varphi})$ consisting of a continuous and Lipschitz map $\tilde{\varphi}: \mathbf{E} \rightarrow \mathbf{E}$ that restricts to a map $\varphi: A \rightarrow A^{\prime}$ through a commutative diagram

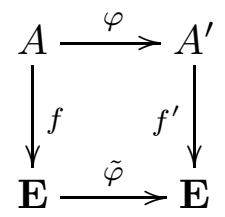

such that $P^{\prime}=\varphi_{*} P$, the pushforward measure given by

$$
\left(\varphi_{*} P\right)_{y}=\sum_{x \in \varphi^{-1}(y)} P_{x}, \quad \forall y \in A^{\prime} .
$$


Thus morphisms in $\mathcal{P}_{\mathbf{E}}$ are subsets of the set of Lipschitz functions of $\mathbf{E}$. The probability distribution $P$ on the finite set $A$ should be thought of as assigning a degree of reliability to the points in the dataset, see the discussion in [BoChYv18]. Points $x \in A$ with a low probability $P_{x}$ should be regarded as errors in the data and discarded in the construction of the associated simplicial complexes.

6.3.5. Vietoris-Rips functors. Given an object $(A, f, P)$ in $\mathcal{P}_{\mathbf{E}}$, we construct a Vietoris-Rips complex $V R(A, f, P)$, obtained by considering, for any choice of an error threshold $\Lambda \in[0,1]$, the set of points

$$
X_{\Lambda}=\left\{x \in f(A) \subset \mathbf{E} \mid P_{x} \geq \Lambda\right\}
$$

and then constructing the Vietoris-Rips complex $V R_{\bullet}\left(X_{\Lambda}, t\right)$ for $t \in \mathbf{R}_{+}^{*}$ where $V R_{n}\left(X_{\Lambda}, t\right)$ is the span of all unordered $(n+1)$-tuples of points $\left(x_{0}, \ldots, x_{n}\right)$ in $X_{\Lambda}$ for which all the pairwise distances satisfy $\operatorname{dist}\left(x_{i}, x_{j}\right) \leq t$. The threshold $\Lambda$ is aimed at discarding a number of outliers of small probability among the data.

Consider then a morphism $(\varphi, \tilde{\varphi})$ in $\mathcal{P}_{\mathbf{E}}$, where $\tilde{\varphi}$ is a Lipschitz function $\tilde{\varphi}: \mathbf{E} \rightarrow$ E with Lipschitz constant $K>0$ and $\varphi: A \rightarrow A^{\prime}$ has $m=\min _{y \in A^{\prime}} \# \varphi^{-1}(y)$. This map sends $X_{\Lambda}$ to $X_{m \Lambda}^{\prime}=\left\{y \in f^{\prime}\left(A^{\prime}\right) \mid P_{y} \geq m \Lambda\right\}$. Moreover, it sends a pair of points $x_{i}, x_{j} \in X_{\Lambda}$ with distance $\operatorname{dist}\left(x_{i}, x_{j}\right) \leq t$ to a pair of points $\tilde{\varphi}\left(x_{i}\right), \tilde{\varphi}\left(x_{j}\right) \in$ $X_{m \Lambda}^{\prime}$ with distance $\operatorname{dist}\left(\tilde{\varphi}\left(x_{i}\right), \tilde{\varphi}\left(x_{j}\right)\right) \leq K t$. Thus, it induces a morphism

$$
V R(\varphi, \tilde{\varphi}): V R_{\bullet}\left(X_{\Lambda}, t\right) \rightarrow V R_{\bullet}\left(X_{m \Lambda}, K t\right)
$$

Consider the set $S=\mathbf{R} \times[0,1]$ with the partial order structure $\leq$ given by the product order $(t, \Lambda) \leq\left(t^{\prime}, \Lambda^{\prime}\right)$ iff $t \leq t^{\prime}$ and $\Lambda \geq \Lambda^{\prime}$ in the natural ordering of the real numbers (with the reverse ordering on $[0,1]$ ). We regard $(S, \leq)$ as a thin category.

6.3.6. Proposition. The assignments $(A, f, P) \mapsto V R(A, f, P)$ and $(\varphi, \tilde{\varphi}) \mapsto$ $\operatorname{VR}(\varphi, \tilde{\varphi})$ as above determine a functor $\mathcal{P}_{\mathbf{E}} \rightarrow \mathcal{M}^{(S, \leq)}$.

Proof. The inclusions $X_{\Lambda} \subset X_{\Lambda}^{\prime}$ for $\Lambda \geq \Lambda^{\prime}$ and the inclusions of the subset of points of $X_{\Lambda}$ with mutual distances bounded above by $t$ in the subset of points with mutual distances at most $t^{\prime}$ for $t \leq t^{\prime}$ induce morphisms

$$
j_{(t, \Lambda),\left(t^{\prime}, \Lambda^{\prime}\right)}: V R_{\bullet}\left(X_{\Lambda}, t\right) \rightarrow V R_{\bullet}\left(X_{\Lambda^{\prime}}, t^{\prime}\right)
$$


for $(t, \Lambda) \leq\left(t^{\prime}, \Lambda^{\prime}\right)$ in the chosen ordering of $S$. Thus, the assignment

$$
V R(A, f, P):(t, \Lambda) \mapsto V R^{(t, \Lambda)}(A, f, P):=V R_{\bullet}\left(X_{\Lambda}, t\right)
$$

determines a functor $(S, \leq) \rightarrow \mathcal{M}$, that is, an object in $\mathcal{M}^{(S, \leq)}$. The morphisms $\operatorname{VR}(\varphi, \tilde{\varphi})$ of Vietoris-Rips complexes described above, for

$$
(\varphi, \tilde{\varphi}) \in \operatorname{Mor}_{\mathcal{P}_{\mathbf{E}}\left((A, f, P),\left(A^{\prime}, f^{\prime}, P^{\prime}\right)\right)}
$$

determine natural transformations $\eta_{(\varphi, \tilde{\varphi})}: V R(A, f, P) \rightarrow V R\left(A^{\prime}, f^{\prime}, P^{\prime}\right)$ of the functors $\operatorname{VR}(A, f, P), \operatorname{VR}\left(A^{\prime}, f^{\prime}, P^{\prime}\right):(S, \leq) \rightarrow \mathcal{M}$, with

$$
\eta_{(\varphi, \tilde{\varphi}),(t, \Lambda)}=V R(\varphi, \tilde{\varphi}): V R_{\bullet}\left(X_{\Lambda}, t\right) \rightarrow V R_{\bullet}\left(X_{m \Lambda}, K t\right)
$$

satisfying

$$
\eta_{(\varphi, \tilde{\varphi}),\left(t^{\prime}, \Lambda^{\prime}\right)} \circ j_{(t, \Lambda),\left(t^{\prime}, \Lambda^{\prime}\right)}=j_{(K t, m \Lambda),\left(K t^{\prime}, m \Lambda^{\prime}\right)} \circ \eta_{(\varphi, \tilde{\varphi}),(t, \Lambda)},
$$

for all $(t, \Lambda) \leq\left(t^{\prime}, \Lambda^{\prime}\right)$ in $(S, \leq)$.

This completes the proof.

In the special case where we fix $\Lambda=0$, hence we consider all the points of $f(A) \subset \mathbf{E}$ regardless of the assigned probabilities, then this construction recovers the usual Vietoris-Rips complexes as objects in $\mathcal{M}^{(\mathbf{R}, \leq)}$ and the functor $V R: \mathcal{P}_{\mathbf{E}} \rightarrow$ $\mathcal{M}^{(\mathbf{R}, \leq)}$ factors through the forgetful functor $\mathcal{P}_{\mathbf{E}} \rightarrow \mathcal{D}_{\mathbf{E}}$ where $\mathcal{D}_{\mathbf{E}}$ is the category of unweighted data sets with objects $(A, f)$ and morphisms given by restrictions of Lifschitz functions of $\mathbf{E}$ (with no conditions on probabilities). The Vietoris-Rips functor then defines a functor $V R: \mathcal{D}_{\mathbf{E}} \rightarrow \mathcal{M}^{(\mathbf{R}, \leq)}$. It is convenient to include the probability data in the construction. We will discuss a more general way of including probability data in the next section.

6.3.7. Dugger's universal model structure. Dugger's construction in [Du01] assigns a universal model category $U(\mathcal{C})$ to a small category $\mathcal{C}$, with the property that functors from $\mathcal{C}$ to a model category factor through $U(\mathcal{C})$. The main idea is that $U(\mathcal{C})$ extends the category $\mathcal{C}$ by formally adjoining homotopy colimits. A factorization of a functor $F: \mathcal{C} \rightarrow \mathcal{M}$, where $\mathcal{M}$ is a model category, through another model category $\tilde{\mathcal{M}}$ with a functor $J: \mathcal{C} \rightarrow \tilde{\mathcal{M}}$ consists of a Quillen pair $L: \tilde{\mathcal{M}} \rightleftarrows \mathcal{M}: R$ and a natural weak equivalence $\eta: L \circ J \rightarrow F$. (For a brief review 
of Quillen pairs see Definition 1.6.3 and Proposition 1.6.4 of [Be18].) The main result of [Du01] shows that given a small category $\mathcal{C}$, there exists a closed model category $U(\mathcal{C})$ with a functor $J: \mathcal{C} \rightarrow U(\mathcal{C})$ such that any functor $F: \mathcal{C} \rightarrow \mathcal{M}$ to a model category $\mathcal{M}$ factors, in the sense recalled above, through $U(\mathcal{C})$.

6.3.8. Proposition. Let $V R: \mathcal{P}_{\mathbf{E}} \rightarrow \mathcal{M}^{(S, \leq)}$ be the Vietoris-Rips functor of Proposition 6.3.6, where $\mathcal{M}^{(S, \leq)}$ has the projective model structure. There is a Quillen pair $L: U\left(\mathcal{P}_{\mathbf{E}}\right) \rightleftarrows \mathcal{M}: R$ and a natural weak equivalence $\eta: L \circ J \rightarrow V R$ that factor the Vietoris-Rips functor through the universal model category $U\left(\mathcal{P}_{\mathbf{E}}\right)$ of the category $\mathcal{P}_{\mathbf{E}}$ of data sets.

Proof. We apply the construction of [Du01] to the category $\mathcal{P}_{\mathbf{E}}$ and we obtain an associated universal model category $U\left(\mathcal{P}_{\mathbf{E}}\right)$. The factorization property discussed above implies that the Vietoris-Rips functor factors through $U\left(\mathcal{P}_{\mathbf{E}}\right)$, when we consider $\mathcal{M}^{(S, \leq)}$ endowed with a model structure. We have seen above that $\mathcal{M}^{(S, \leq)}$ always supports the projective model structure, seen as the category of functors $\mathcal{F}((S, \leq), \mathcal{M})$.

\section{PERSISTENCE AND $\Gamma$-SPACES}

7.1. Setup. We discuss in this section how to adapt to the context of persistent topology another important homotopy-theoretic construction: Segal's $\Gamma$-spaces. We consider a (slightly modified) setting developed in [Mar18] that incorporates probabilistic data in the construction of Segal's $\Gamma$-spaces. They replace the finite probability distributions considered in the previous section in the context of persistent topology of databases.

Our point of view here is somewhat different from the one proposed in [Mar18] and more closely related to our discussion of the Vietoris-Rips functor in the previous section. The point we want to stress here is that the Vietoris-Rips functor, as we described it above, can be generalized using Segal's $\Gamma$-spaces ([Se74]). A $\Gamma$ space is a functor $F: \Gamma^{0} \rightarrow \Delta \mathcal{S}_{*}$ from the category of pointed finite sets to pointed simplicial sets. In particular, Segal showed in [Se74] that to a category $\mathcal{C}$ with a categorical sum and a zero object one can associate a $\Gamma$-space obtained by assigning to a finite pointed set $(X, \star)$ the nerve $\mathcal{N} \Sigma_{\mathcal{C}}(X, \star)$ of the category $\Sigma_{\mathcal{C}}(X, \star)$ of summing functors $\Phi: P(X, \star) \rightarrow \mathcal{C}$, where $P(X, \star)$ is the category with objects the pointed subsets of $X$ and morphisms the pointed inclusions, and the summing functors satisfy $\Phi_{X, \star}(S) \oplus \Phi_{X, \star}\left(S^{\prime}\right) \simeq \Phi_{X, \star}\left(S \cup S^{\prime}\right)$ for all $S, S^{\prime} \in P(X, \star)$ with $S \cap S^{\prime}=\{\star\}$. A map of pointed sets $f:(X, \star) \rightarrow\left(Y, \star^{\prime}\right)$ induces on summing functors a trans- 
formation $\Sigma_{\mathcal{C}}(f): \Sigma_{\mathcal{C}}(X, \star) \rightarrow \Sigma_{\mathcal{C}}\left(Y, \star^{\prime}\right)$ given by $\Sigma_{\mathcal{C}}(f)\left(\Phi_{X, \star}\right)(S)=\Phi_{X, \star}\left(f^{-1}(S)\right)$, for all $S \in P\left(Y, \star^{\prime}\right)$.

As in the previous section, we consider a category of databases, identified with finite sets endowed with probabilities, embedded in an ambient metric space (a large dimensional Euclidean space $\mathbf{E}$ ). To adapt the setting to the pointed case required for the $\Gamma$-space formalism, we consider here pointed sets, so we work with the category $\mathcal{P}_{\mathbf{E}, *}$ whose objects are triples $((X, \star), f, P)$ of a finite pointed set $(X, \star)$ with a probability measure $P$ and an embedding $f: X \hookrightarrow \mathbf{E}$ with $f(\star)=0$ the origin, in the Euclidean space $\mathbf{E}$. Morphisms are pairs $(\varphi, \tilde{\varphi})$ of a measurepreserving pointed map $\varphi$ of finite sets and a Lipshitz self-map $\tilde{\varphi}$ of $\mathbf{E}$ that fixes the origin, which restricts to $\varphi$ on the images under the embeddings.

Following the construction of the Vietoris-Rips complex in the previous section, we consider the poset $(S, \leq)$ with $S=\mathbf{R}_{+} \times[0,1]$ with the natural order on $t \in \mathbf{R}_{+}$ and the reverse order on $\Lambda \in[0,1]$. We then have the following generalizations of the Vietoris-Rips functor with values in $\Delta \mathcal{S}^{(S, \leq)}$.

7.2. Proposition. Any $\Gamma$-space $F_{\mathcal{C}}: \Gamma^{0} \rightarrow \Delta \mathcal{S}_{*}$ determines a functor

$$
\tilde{F}_{\mathcal{C}}: \mathcal{P}_{\mathbf{E}, *} \rightarrow \Delta \mathcal{S}_{*}^{(S, \leq)}
$$

Proof. Start with a $\Gamma$-space $F_{\mathcal{C}}: \Gamma^{0} \rightarrow \Delta \mathcal{S}_{*}$ associated as above to a category $\mathcal{C}$ with sum and zero object.

We obtain from it a functor $\tilde{F}_{\mathcal{C}}: \mathcal{P}_{\mathcal{E}, *} \rightarrow \Delta \mathcal{S}_{*}^{(S, \leq)}$ in the following way. For $s=(t, \Lambda) \in S$ we denote by $\left(X_{t, \Lambda}, \star\right)$ the finite pointed set $\{\star\} \cup Y_{t, \Lambda}$ where $Y=X \backslash\{\star\}$ and $Y_{t, \Lambda}$ is the subset of all the non-marked points of $X$ with mutual distances $d_{\mathbf{E}}\left(f(x), f\left(x^{\prime}\right)\right) \leq t$ and with probabilities $P_{x} \geq \Lambda$, as in the previ-

ous section. We can then set $\tilde{F}_{\mathcal{C}}((X, \star), f, P)(s)=F_{\mathcal{C}}\left(X_{s}\right)$. For $s \leq s^{\prime}$ in $S$ we have an inclusion $X_{s} \hookrightarrow X_{s^{\prime}}$. This induces a transformation as above $\Sigma_{\mathcal{C}}\left(X_{s}\right) \rightarrow$ $\Sigma_{\mathcal{C}}\left(X_{s^{\prime}}\right)$ on summing functors, hence a map $F_{\mathcal{C}}\left(X_{s}\right) \rightarrow F_{\mathcal{C}}\left(X_{s^{\prime}}\right)$, hence we obtain a functor $\tilde{F}_{\mathcal{C}}((X, \star), f, P):(S, \leq) \rightarrow \Delta \mathcal{S}_{*}$. Morphisms $(\varphi, \tilde{\varphi}):((X, \star), f, P) \rightarrow$ $\left(\left(X^{\prime}, \star^{\prime}\right), f^{\prime}, P^{\prime}\right)$ in $\mathcal{P}_{\mathbf{E}, *}$ induce by restriction maps $\varphi_{s}: X_{s} \rightarrow X_{s^{\prime}}$ and corresponding maps on summing functors $\Sigma_{\mathcal{C}}\left(X_{s}\right) \rightarrow \Sigma_{\mathcal{C}}\left(X_{s^{\prime}}\right)$ as above.

\section{FURTHER DIRECTIONS}

8.1. Large scale geometry. The idea of studying properties of metric spaces at large scales was introduced by Gromov [Gro81] in the context of groups of poly- 
nomial growth. It was later developed into a broad framework for coarse geometry and large scale geometry, see [NoYu12], [Roe03]. Certain (co)homology functors for coarse geometry have been introduced in [Roe93], see also Chapter 7 of [NoYu12]. In particular, the coaresening of homology theories described in Section 7.5 of [NoYu12] is based on the same notion of scale-dependent Vietoris-Rips complexes that we discussed above in the setting of persistent homology. Thus, we expect that the approach to persistence in terms of Nori diagrams that we advocate in this paper should be applicable also to the context of coarse geometry. It would be interesting to compare it with the axiomatic formulations of coarse homology given in [Mitch01]. Among the interesting current problems in coarse geometry are various topological and geometric rigidity conjectures (see Chapter 8 of [NoYu12]), which can be approached via index theory methods, developed in the coarse geometry setting in [Roe93]. It would be interesting to investigate whether index theory in the coarse geometry context can be formulated in terms of a more "motivic" view of large scale geometry and coarse homology.

8.2. Cantor-like barcodes and fractality. It was obsevred in [KapVa04], [Pre11] how ind-pro objects over a category behave as Cantor-like objects. This property was used in [Li11] to model algebro-geometrically, in terms of ind-pro varieties, the energy-crystal momentum dispersion relation for Harper and almost Mathieu operators with irrational parameters, replacing the ordinary Bloch variety by an ind-pro object, which parallels the occurrence of the Hofstadter butterfly at the level of the spectrum, with its Cantor set fractal structure. The density of states and the spectral functions are obtained in [Li11] as periods on this "fractal-like" ind-pro version of the Bloch variety.

Within the context of this paper, one can consider the possibility of extending the persistence structures and associated barcode diagrams to a larger class of objects obtained as limits of finite type objects in $\operatorname{Vec}^{(\mathbf{R}, \leq)}$ taken in such a way that the associated barcode diagrams become Cantor sets. It would be interesting to investigate whether a larger class of physical models similar to the algebrogeometric formulation of Harper operators given in [Li11] could be analyzed in terms of such limits of persistent homologies.

8.3. Khovanov homology and thin poset (co)homologies. In [Khov00] Khovanov constructed a categorification of the Jones polynomial in the form of a chain complex of graded vector spaces and corresponding homology whose graded Euler characteristic is the Jones polynomial. The chain complex is constructed by assigning to a knot or link diagram with $N$ cossings the poset given by the $N$-cube 
and a functor from this thin category to the category of graded vector spaces. The graded vector space assigned to a vertex of the cube corresponds to a smoothing of the link where all the crossings are eliminated resulting in a union of $k$ planar closed curves, and the graded dimension of the associated vector space depends on $k$ and on the degree of the vertex, see [BarNat02] for more details.

A generalization of this construction is given in [Chand18], where a chain complex and a Khovanov-type cohomology $H^{*}(F, S, \mathcal{A})$ are associated to any functor $F$ : $(S, \leq) \rightarrow \mathcal{A}$ from a poset to an abelian category, where the poset has a "thinness" property described as follows. One requires the existence of a grading $r: S \rightarrow \mathbf{N}$ with $r(x) \leq r(y)$ for $x \leq y$, such that any pair $x, y \in S$ with $x \leq y$ for which there is no $z \in S$ with $x<z<y$ should have $r(y)=r(x)+1$ and when $r(y)=r(x)+2$ the set $\{z \in S: x<z<y\}$ consists of exactly two elements. For posets $(S, \leq)$ that satisfy this thinness property, given a functor $F:(S, \leq) \rightarrow \mathcal{A}$ one constructs a chain complex with $C^{k}(F, S, \mathcal{A})=\oplus_{r(x)=k} F(x)$ and $\delta^{k}=\sum c(x, y) F(x \leq y)$, where the sum is over all pairs $x, y$ with $x \leq y$ such that there is no $z$ with $x<z<y$, and $c$ is a "balanced coloring". This is a $\{ \pm 1\}$ valued function on the set of pairs as above, with the property that it has an odd number of -1 's on each "diamond" set $\{z \in S: x \leq z \leq y\}$ with $r(y)=r(x)+2$. The thinness property of the poset and the balanced coloring property ensure that $\delta^{2}=0$ so that one obtains a chain complex. This general formalism is very suitable for introducing persistent versions of Khovanov homology and related constructions and investigating the topological information about knots and links that these persistent functors would capture.

Acknowledgment. We thank Jack Morava for suggesting the question of model structures for persistent homology discussed in Section 6. The second author is partially supported by NSF grant DMS-1707882, by NSERC Discovery Grant RGPIN2018-04937 and Accelerator Supplement Grant RGPAS-2018-522593, by the FQXi grant FQXi-RFP-1 804, and by the Perimeter Institute for Theoretical Physics.

\section{References}

[An04] Y. André. Une introduction aux motives (motifs purs, motifs mixtes, périodes.) Panoramas et Synthèses, vol. 17. Société Mathématique de France, Paris, 2014.

[Ar13] D. Arapura. An abelian category of motivic sheaves. Adv. Math., 233, 2013, pp. 135-195. arXiv:0801.0261 
[Bar94] S.A. Barannikov, The Framed Morse complex and its invariants, Adv. Soviet Math. Vol.21 (1994) 93-115.

[BarNat02] D. Bar-Natan, On Khovanov's categorification of the Jones polynomial, Algebraic \& Geometric Topology, 2 (2002) 337-370. arXiv:math.QA/0201043

[BGSV90] A. Beilinson, A. Goncharov, V. Schechtman, A. Varchenko. Aomoto dilogarithms, mixed Hodge structures and motivic cohomology of pairs of triangles on the plane. In "The Grothendieck Festschrift", Vol. I, pp.135-172, Progr. Math., Vol. 86, Birkhäuser, 1990.

[Be18] J. Bergner, The homotopy theory of $(\infty, 1)$-categories. Cambridge University Press, 2018.

[BluLes17] A. Blumberg, M. Lesnick, Universality of the homotopy interleaving distance, arXiv:1705.01690

[BoChYv18] J. Boissonnat, F. Chazal, M. Yvinec. Geometric and topological inference. Cambridge University Press, 2018.

[Br14a] M. Brion. On algebraic semigroups and monoids. In "Algebraic monoids, group embeddings, and algebraic combinatorics", pp.1-54, Fields Inst. Commun., 71, Springer, 2014.

[Br14b] M. Brion. On algebraic semigroups and monoids, II. Semigroup Forum 88 (2014) no. 1, pp. 250-272.

[BuSc14] P. Bubenik, J. Scott. Categorification of persistent homology. Discrete and Computational Geometry, 51 (3), 2014, pp. 600-627.

[BuSiSc15] P. Bubenik, V. de Silva, J. Scott. Metrics for generalised persistence modules. Foundations of Computational Math., vol. 15 (2015), issue 6, pp. 15011531.

[Car09] G. Carlsson, Topology and data. Bull. Amer. Math. Soc. (N.S.) 46, no. 2, 2009, pp. 255-308.

[Chand18] A. Chandler, Thin posets and homology theories, preprint, 2018

https://alexchandler.wordpress.ncsu.edu/preprints/

[Du01] D. Dugger, Universal homotopy theories. Adv. Math., Vol.164 (2001), N.1, pp. 144-176.

[EdHar10] H. Edelsbrunner, J. Harer. Computational topology. American Mathematical Society, 2010. 
[EPY03] D. Eisenbud, S. Popescu, S. Yuzvinsky. Hyperplane arrangement cohomology and monomials in the exterior algebra. Trans. Amer. Math. Soc. 355 (2003), no. 11, 4365-4383.

[GeMa03] S. Gelfand, Yu. Manin. Methods of homological algebra. 2nd Edition. Springer 2003, xvii + 372 pp.

[GoJa] P. Goerss, R. Jardine. Simplicial homotopy theory. Birkhäuser 1999.

[Gro81] M. Gromov, Groups of polynomial growth and expanding maps, Publ. Math. IHES, 53 (1981) 53-73.

[Hat02] A. Hatcher. Algebraic Topology. Cambridge UP, 2002.

[Hi03] Ph. Hirschhorn. Model categories and their localizations. American Mathematical Society, 2003.

[Ho98] M. Hovey, Model categories, Mathematical Surveys and Monographs, Vol. 63, American Math- ematical Society, 1998.

[HuM-S17] A. Huber, St. Müller-Stach. Periods and Nori motives. With contributions by Benjamin Friedrich and Jonas von Wangenheim. Springer, 2017, xxiii+372 pp.

[KapVas04] M. Kapranov, E. Vasserot, Vertex algebras and the formal loop space, Publ. Math. IHES 100 (2004) 209-269.

[KaSch06] M. Kashiwara, P. Schapira. Categories and sheaves. Springer 2006, x +497 pp.

[KaSch17] M. Kashiwara, P. Schapira. Persistent homology and microlocal sheaf theory. arXiv:1705.00955, 30 pp.

[Khov00] M. Khovanov, A categorification of the Jones polynomial, Duke Math. J. 101 (2000) no. 3, 359-426. arXiv:math.QA/9908171

[Li11] D. Li, The algebraic geometry of Harper operators, J. Phys. A 44 (2011), no. 40, 405204, 27 pp.

[Mar18] M. Marcolli, Gamma Spaces and Information. J. Geom. Phys. 140 (2019), 26-55. arXiv:1807.05314.

[MaBo07] Yu. Manin, D. Borisov. Generalized operads and their inner cohomomorhisms . In: Geometry and Dynamics of Groups and spaces (In memory of Aleksader Reznikov). Ed. by M. Kapranov et al. Progress in Math., vol. 265. Birkhäuser, Boston, 2007, pp. 247-308. arXiv:math.CT/0609748 
[Mitch01] P.D. Mitchener, Coarse homology theories. Algebr. Geom. Topol. 1 (2001) 271-297.

[Mi86] H. Mitsch. A natural partial order for semigroups. Proc. American Math. Soc., Vol. 97 (1986) No. 3, 384-388.

[Mu06] D. Murfet. Abelian Categories. Preprint, 2006

http://therisingsea.org/notes/AbelianCategories.pdf

[Na80] K. Nambooripad. The natural partial order on a regular semigroup. Proc. Edinburgh Math. Soc. 23 (1980), pp. 249-260.

[NoYu12] P.W. Nowak, G. Yu, Large scale geometry, European Mathematical Society, 2012. xiv+189 pp.

[Pre11] L. Previdi, Locally compact objects in exact categories, Internat. J. Math. 22 (2011) no. 12, 1787-1821.

[Roe03] J. Roe, Lectures on coarse geometry, University Lecture Series, Vol. 31, American Mathematical Society, 2003. viii+175 pp.

[Roe93] J. Roe, Coarse cohomology and index theory on complete Riemannian manifolds. Mem. Amer. Math. Soc. 104 (1993) no. 497, x+90 pp.

[SchSh00] S. Schwede, B. Shipley. Algebras and modules in monoidal model categories. Proc. London Math. Soc. (3) 80 (2000), no. 2, pp. 491-511.

arXiv:math/9801082

[Se74] G. Segal. Categories and cohomology theories. Topology, Vol.13 (1974) 293-312.

[Za05] A. Zomorodian. Topology for computing. Cambridge University Press, 2005. 